\title{
Fuzzy Global Stability Analysis of the Dynamics of Malaria with Fuzzy Transmission and Recovery Rates
}

\author{
Yves Tinda Mangongo, Joseph-Désiré Kyemba Bukweli, Justin Dupar Busili Kampempe \\ Department of Mathematics and Computer Science, Faculty of Sciences, University of Kinshasa, Kinshasa, D.R. Congo \\ Email: yves.mangongo@unikin.ac.cd, desire.bukweli@unikin.ac.cd, Justin-dupar.kampempe@unikin.ac.cd
}

How to cite this paper: Mangongo, Y.T., Bukweli, J.-D.K. and Kampempe, J.D.B. (2021) Fuzzy Global Stability Analysis of the Dynamics of Malaria with Fuzzy Transmission and Recovery Rates. American Journal of Operations Research, 11, 257-282.

https://doi.org/10.4236/ajor.2021.116017

Received: September 9, 2021

Accepted: October 23, 2021

Published: October 26, 2021

Copyright $\odot 2021$ by author(s) and Scientific Research Publishing Inc. This work is licensed under the Creative Commons Attribution International License (CC BY 4.0).

http://creativecommons.org/licenses/by/4.0/

(c) (i) Open Access

\begin{abstract}
In this paper, fuzzy techniques have been used to track the problem of malaria transmission dynamics. The fuzzy equilibrium of the proposed model was discussed for different amounts of parasites in the body. We proved that when the amounts of parasites are less than the minimum amounts required for disease transmission $\left(v \leq v_{\min }\right)$, we reach the model disease-free equilibrium. Using Choquet integral, the fuzzy basic reproduction number through the expected value of fuzzy variable was introduced for the fuzzy Susceptible, Exposed, Infected, Recovered, susceptible-Susceptible, Exposed and Infected (SEIRS-SEI) malaria model. The fuzzy global stabilities were introduced and discussed. The disease-free equilibrium $Y^{0}$ is globally asymptotically stable if $v \leq v_{\min }$ or if the basic reproduction number is less than one $\left(\mathcal{R}_{0}(v) \leq 1\right)$. When $v>v_{\text {min }}$ and $\mathcal{R}_{0}(v)>1$, there exists a co-existing endemic equilibrium which is globally asymptotically stable in the interior of feasible set $\Omega$. Finally, the numerical simulation has been done for showing the effectiveness of our analytical results.
\end{abstract}

\section{Keywords}

Malaria, Fuzzy Analysis, Fuzzy Basic Reproduction Number, Fuzzy Variable, Credibility Measure, Fuzzy Equilibrium, Fuzzy Global Stability

\section{Introduction}

Malaria, a mosquito-borne disease, is one of the oldest diseases studied in mathematical approaches. It is one the public health problem across the world. In 2020, WHO observed a real decreasing of malaria cases. The total number of malaria cases has fallen from 238 million in 2000 to 229 million in 2019 [1]. 
During that period, the population of Sub-Saharan Africa, which comprises more than $90 \%$ of the global burden of malaria had increased from 665 million to over a billion people [1]. Malaria is transmitted during a bloodmeal by an infected female anophele mosquito. Malaria is developed in two stages [2]. Firstly in mosquitoes (vectors), the parasite enters during a bloodmeal of a susceptible mosquito to an infected human. After the parasite is developed, the mosquito becomes contagious between 10 to 16 days [3]. Secondly in humans (hosts), the parasite enters during a bloodmeal of an infected mosquito to susceptible human.

The mathematical study of infectious diseases is important and a more realistic tool for a better understanding of their evolution, stability, control and for identifying the influential parameters in the spread of the disease [4] [5]. Since the first mathematical model of malaria in 1910 with Ronald Ross [6], many studies have been done in malaria modeling. Some of them included the environmental, climatic, seasonal and periodic aspects [7] [8] [9] [10] [11]. Others are interesting in vertical transmission and relapse [12] [13] [14].

However, most of these models used a deterministic approach with constant parameters. For example, they assumed that the contact transmission and recovery rates are constant. These assumptions are not realistic in the context of malaria transmission. The parameters such as contact and recovery rates are uncertain in reality. They depend on the amount of parasites (parasitic viral load) in the blood cells [15] [16]. In addition, the classical deterministic mathematical models which describe malaria do not incorporate the high degree of subjectivity involved in modeling. In order to describe the transmission dynamics of an infectious disease (malaria for this study) in a more realistic way, several models that incorporate some subjectivity and heterogeneity by the use of fuzzy theory introduced by Zadeh [17], have been studied and proposed. L. C. de Barros et al. in 2003 studied an SI epidemiological model with a fuzzy transmission parameter. In this model, they consider different degrees of infectivity of contact rate. Madhu Jain et al. [18] studied the transmission dynamics of malaria in human host using a neuro-fuzzy approach. Hassan Zarei et al. [19] used fuzzy theory in the modeling and control of HIV infection. They proposed a fuzzy mathematical model of HIV infection consisting of a linear fuzzy differential equations (FDEs) system describing the ambiguous immune cells level and the viral load which are due to the intrinsic fuzziness. Mondal et al. [20] studied the SIS epidemiological model by considering the disease transmission parameter and treatment control parameter as fuzzy number. Belinda O. Emokhare and Emadomi M. Igbape [21] used a fuzzy logic approach of Ebola Hemorrhagic Fever. Renu Verma et al. [22] proposed a fuzzy SIR epidemic model. In their work, they assumed that the transmission rate, the recovery and disease-induced rate are fuzzy numbers. More recently in 2020, G. Bhuju et al. used a fuzzy approach to track the problem of Dengue transmission dynamics in Nepal using the SEIR-SEI scheme. In their work, they assumed that the transmission and the recovery rates 
are fuzzy numbers. Following this way, in this paper, we present a SEIRS-SEI model for transmission of malaria dynamics in fuzzy environment. It was proven that malaria transmission occurs when a certain amount of parasites (parasitic viral load) is reached. In addition, a human is recovered from malaria if the amount of parasites in the body is less than a certain minimum amount of parasites.

In this paper, we assume that the transmission and recovery rates are fuzzy variables (depend on the amount of parasites in the blood cells). We have used the expected value of fuzzy variable introduced by Baoding Liu and Yian-Kui [23] to compute the fuzzy basic reproduction number. We introduce the fuzzy analysis of the equilibrium of model, we compute and analyze the fuzzy basic reproduction number. We introduce and derive the fuzzy global stabilities of the proposed fuzzy model by the use of matrix-theoretical and graph-theoretical methods introduced by Z. Shuai and P. van den Driessche [24].

The paper is organized as follows: in Section 2, we present a summary of fuzzy concepts which will be used in the sequel of this paper. We define a fuzzy variable, credibility measure and fuzzy expected value. In Section 3, we formulate the new fuzzy SEIRS-SEI model for malaria transmission dynamics and we define the assumed fuzzy parameters. Section 4 introduces the fuzzy equilibrium of the model, presents the fuzzy basic reproduction number of the proposed fuzzy model and introduces the fuzzy global stabilities of the proposed model. Section 5 gives some numeric results and discussions. Finally, section 6 is concentrated to some concluding remarks.

\section{Preliminaries}

Let $X$ be a nonempty set. A fuzzy set $\xi$ of $X$ is defined as a set of all pairs $(x, \mu(x)) \in X \times[0,1]$. The function $\mu$, defined in $X$ is called "membership function" and $\mu(x)$ indicates the belonging degree of $x$ in the fuzzy set $\xi$ [25] [26]. The fuzzy set $\xi$ will be a fuzzy number if:

- $X$ is a set of real numbers and there exists at least $x_{0} \in \mathbb{R}$ such that $\mu\left(x_{0}\right)=1$,

- $\mu$ is upper semi-continuous on $\mathbb{R}$ and the support of $\xi$ is a compact set,

- $\xi$ is a convex fuzzy set. That is, $\mu(\lambda x+(1-\lambda) y) \geq \min \{\mu(x), \mu(y)\}$, for all $x, y \in \mathbb{R}$ and $\lambda \in[0,1]$.

A fuzzy variable is defined as a measurable function from a credibility space $(X, \mathcal{P}(X), C r)$ to the set of real numbers. Where $\mathcal{P}(X)$ is the power set of $X$ and $C r$ the credibility measure. For any real number $r$, it's defined as:

$$
\operatorname{Cr}\{\xi \leq r\}=\frac{1}{2}\left(\sup _{t \leq r} \mu(t)+1-\sup _{t>r} \mu(t)\right) \in[0,1] \text {. }
$$

Note that the credibility measure is the average of possibility and necessity measures introduced by L. Zadeh [27], defined respectively by:

$$
\operatorname{Poss}(\{\xi \leq r\})=\sup _{t \leq r} \mu(t)
$$

and 


$$
\operatorname{Nec}(\{\xi \leq r\})=1-\sup _{t>r} \mu(t) .
$$

None of these two measures is self-dual. That reason justified the introduction of the credibility measure by B. Liu and Y.-K. Liu [23] [28] [29]. The self-duality property is very important in order to control the behavior of a system. From here a fuzzy number can be considered as a particular case of a fuzzy variable. In order to use the defuzzification process to obtain some crisps values (real numbers) which represents a fuzzy number, we use the Expected Value of Fuzzy Variable introduced by B. Liu and Y.-K. Liu, denoted by $E[\xi]$ and defined as follows:

$$
E[\xi]=\int_{0}^{+\infty} \operatorname{Cr}\{\xi \geq r\} \mathrm{d} r-\int_{-\infty}^{0} \operatorname{Cr}\{\xi \leq r\} \mathrm{d} r,
$$

provided that at least one of these integrals is finite. The expected value of a trapezoidal fuzzy variable denoted by $\xi=(a, b, c, d)$ is given by:

$$
E[\xi]=\int_{0}^{+\infty} \operatorname{Cr}\{\xi \geq r\} \mathrm{d} r-\int_{-\infty}^{0} \operatorname{Cr}\{\xi \leq r\} \mathrm{d} r=\frac{a+b+c+d}{4},
$$

and the expected value of a triangular fuzzy variable denoted by $\xi=(a, b, c)$ is given by:

$$
E[\xi]=\int_{0}^{+\infty} \operatorname{Cr}\{\xi \geq r\} \mathrm{d} r-\int_{-\infty}^{0} \operatorname{Cr}\{\xi \leq r\} \mathrm{d} r=\frac{a+2 b+c}{4} .
$$

Since, $E[\xi]$ is a real, in this paper it will be used to defuzzify some of the fuzzy parameters which will be used.

\section{The Fuzzy SEIRS-SEI Model for Malaria Transmission}

In this model, we use two classical schemes known in the Literature. For human population we use the classical SEIRS scheme and for mosquitoes population, we use the SEI scheme. The model describes fuzzy interaction between individuals (humans and mosquitoes) in these two populations.

We consider the different degrees of susceptibility and infectivity of malaria among populations. Considering the heterogeneity of population, in this work, we assume that the contact transmission rate $\beta_{h}$ (chance that in one contact between a susceptible human and an infected mosquito results to an infection of susceptible human) and recovery rate $\gamma_{h}$ are fuzzy variables. To describe the amount of parasites (parasitic viral load) $v$ in the transmission rate $\beta_{h} \equiv \beta_{h}(v)$, we use the membership function introduced firstly by L.C. de Barros [5] and used by G. Bhuju et al. [4] and Renu Verma et al. [22] defined as:

$$
\beta_{h}(v)= \begin{cases}0, & \text { if } v \leq v_{\min } \\ \frac{v-v_{\min }}{v_{M}-v_{\min }}, & \text { if } v_{\min }<v<v_{M} \\ 1, & \text { if } v_{M} \leq v \leq v_{\max }\end{cases}
$$

The membership function defined on 7 can be interpreted as: the transmission chance is negligible if the amount of parasites is less than or equal to $v_{\min }$. That 
is, there is a certain amount of parasites needed to cause malaria transmission. For a certain amount of parasites $v \in\left[v_{M}, v_{\max }\right]$, there is a maximum chance of malaria transmission. Addition, for some amount of parasites between $v_{\min }$ and $v_{M}$, there is a chance that the malaria transmission occurs. The membership function of the transmission rate $\beta(v)$ is represented on Figure 1. Recovering from malaria is a fuzzy variable. The recovery occurs when the amount of parasites is reducing in the blood cells. As said above, we consider the recovery rate $\gamma_{h}$ as a fuzzy variable depending on the amount of parasites $v$. In this paper, we use the fuzzy recovery rate introduced by Renu Verma et al. [22] and modified by G. Bhuju et al. [4] as:

$$
\gamma_{h}(v)=\frac{\gamma_{0}-1}{v_{\max }} v+1, \text { if } 0 \leq v \leq v_{\max }
$$

where $0<\gamma_{0}<1$ is the human recovery rate when we reach a maximum amount of parasites in the blood cells.

The amount of parasites is different to each group of humans. Therefore, making the model more realistic, we consider only the human individuals in a given group $\mathrm{V}$. With classification (weak, medium and strong) given by an expert, $\mathrm{V}$ can be seen as a linguistic variable with membership function $\Gamma(v)$ defined by L. C. Barros [5] as:

$$
\Gamma(v)= \begin{cases}0, & \text { if } v<\bar{v}-\delta \\ \frac{\delta+v-\bar{v}}{\delta}, & \text { if } \bar{v}-\delta \leq v \leq \bar{v} \\ \frac{\delta+\bar{v}-v}{\delta}, & \text { if } \bar{v}<v \leq \bar{v}+\delta \\ 0, & \text { if } v>\bar{v}+\delta\end{cases}
$$

The parameter $\bar{v}$ is a central value and $\delta$ gives the dispersion of each one of the fuzzy sets assumed by V. Its graphic representation is given on Figure 2 which has a triangular sharp.

The fuzzy incidence rate of mosquitoes to human is given by $\lambda_{h}(v)=\beta_{h}(v) \frac{I_{m}}{N_{h}}$.

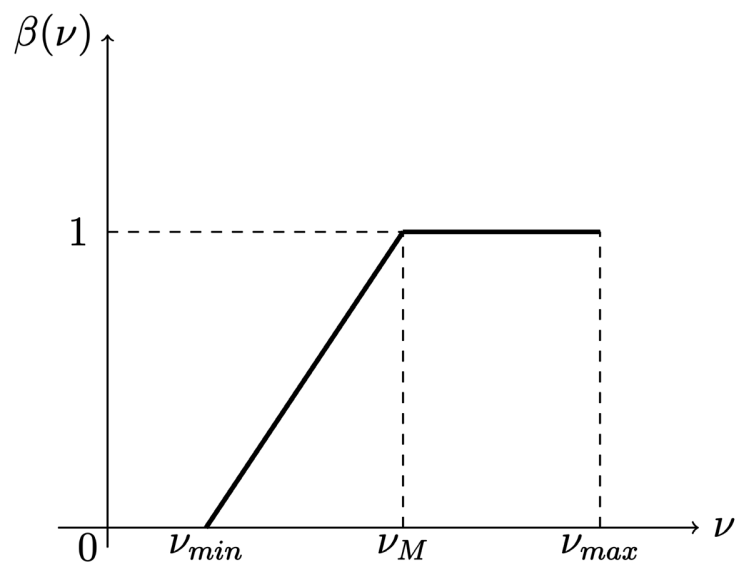

Figure 1. Membership function of $\beta(v)$. 
The incidence rate of humans to mosquitoes is given by $\lambda_{m}=\beta_{m} \frac{I_{h}}{N_{h}}$, where $\beta_{m}$ represents the parasite transmission rate of humans to mosquitoes. Then, the fuzzy SEIRS-SEI malaria model describing on Figure 3 is given by the following seven ordered system of ordinary differential equations (ODEs) with fuzzy parameters.

$$
\begin{gathered}
\frac{\mathrm{d} S_{h}}{\mathrm{~d} t}=\mu N_{h}-\beta_{h}(v) \frac{I_{m}}{N_{h}} S_{h}-\mu S_{h}+\theta_{h} R_{h} \\
\frac{\mathrm{d} E_{h}}{\mathrm{~d} t}=\beta_{h}(v) \frac{I_{m}}{N_{h}} S_{h}-\left(\sigma_{h}+\mu\right) E_{h} \\
\frac{\mathrm{d} I_{h}}{\mathrm{~d} t}=\sigma_{h} E_{h}-\left(\mu+\delta+\gamma_{h}(v)\right) I_{h} \\
\frac{\mathrm{d} R_{h}}{\mathrm{~d} t}=\gamma_{h}(v) I_{h}-\left(\mu+\theta_{h}\right) R_{h}
\end{gathered}
$$

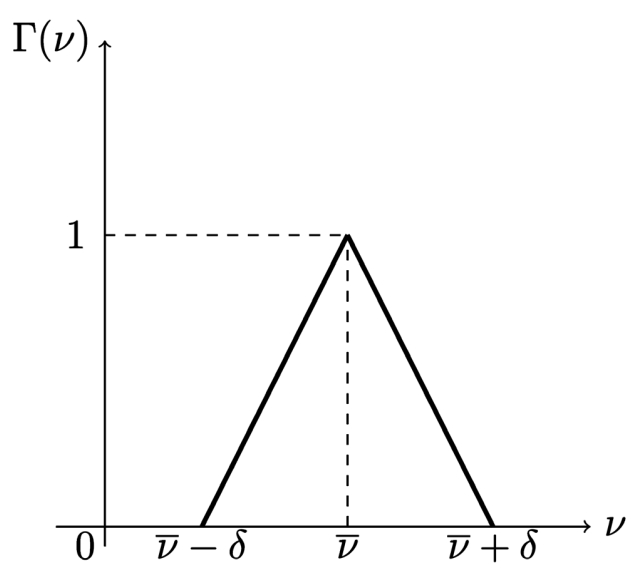

Figure 2. Membership function of $\Gamma(v)$.

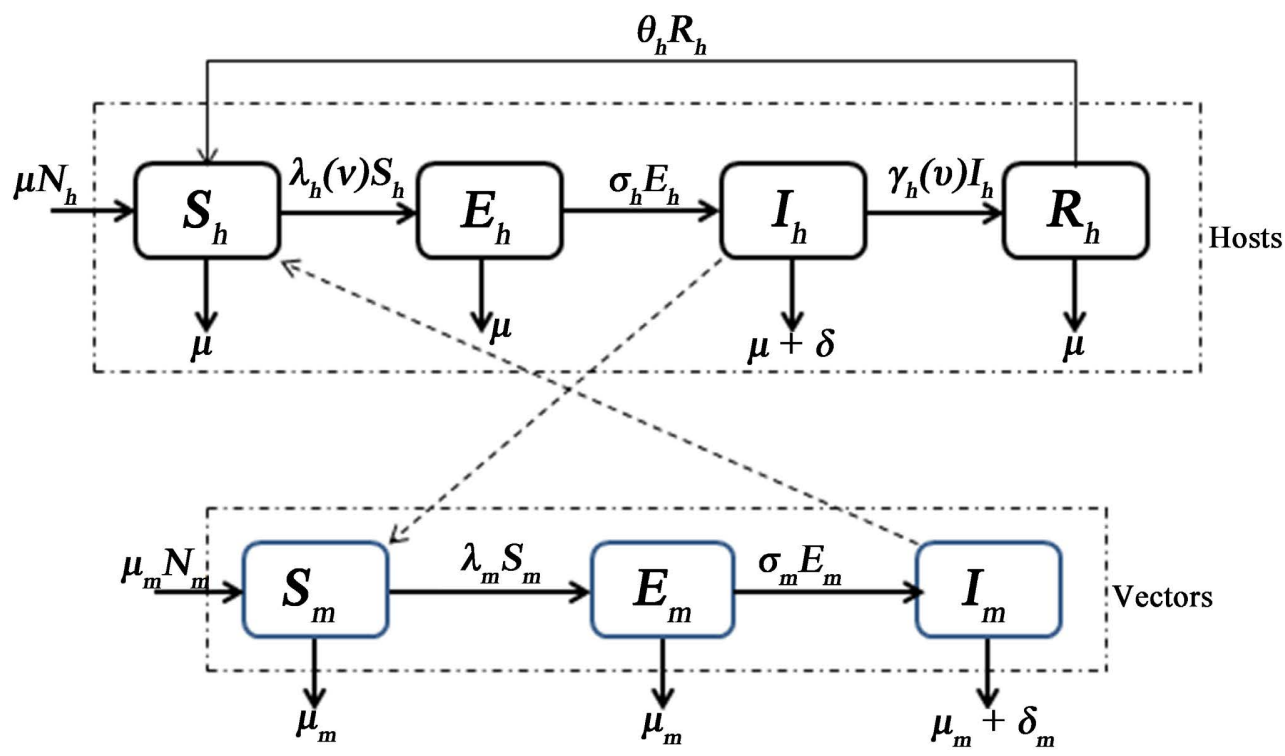

Figure 3. The fuzzy flow diagram. 


$$
\begin{aligned}
\frac{\mathrm{d} S_{m}}{\mathrm{~d} t} & =\mu_{m} N_{m}-\beta_{m} \frac{I_{h}}{N_{h}} S_{m}-\mu_{m} S_{m} \\
\frac{\mathrm{d} E_{m}}{\mathrm{~d} t} & =\beta_{m} \frac{I_{h}}{N_{h}} S_{m}-\left(\sigma_{m}+\mu_{m}\right) E_{m} \\
\frac{\mathrm{d} I_{m}}{\mathrm{~d} t} & =\sigma_{m} E_{m}-\left(\mu_{m}+\delta_{m}\right) I_{m}
\end{aligned}
$$

The system (10)-(16) is solved under the non negative initial conditions:

$$
Y(0)=\left(S_{h}(0), E_{h}(0), I_{h}(0), R_{h}(0), S_{m}(0), E_{m}(0), I_{m}(0)\right) \in \Omega \subseteq \mathbb{R}_{+}^{7} .
$$

The total population of human at each time is given by:

$$
N_{h}(t)=S_{h}(t)+E_{h}(t)+I_{h}(t)+R_{h}(t),
$$

and the total population of mosquitoes at each time is managed by:

$$
N_{m}(t)=S_{m}(t)+E_{m}(t)+I_{m}(t) .
$$

We assume equal the recruitment and natural death rates of human population at the rate $\mu$. The recruitment and natural death rates of mosquitoes are also assumed to be equal to $\mu_{m}$. Parameters $\sigma_{h}$ and $\sigma_{m}$ represent the latent rates of both humans and mosquitoes respectively. Parameters $\delta$ and $\delta_{m}$ denote the malaria-induced death rate and the parasite-induced death rate respectively. Below, we prove the well-posedness of model, we prove the existence, non negativity and the boundness of solution of the system (10)-(16).

Suppose that

$$
\begin{aligned}
Y(t) & =\left(S_{h}(t), E_{h}(t), I_{h}(t), R_{h}(t), S_{m}(t), E_{m}(t), I_{m}(t)\right) \in \Omega \subseteq \mathbb{R}_{+}^{7} \\
g: \Omega & \rightarrow \Sigma \subseteq \mathbb{R}_{+}^{7} ; g(Y(t))=\frac{\mathrm{d} Y(t)}{\mathrm{d} t} . \text { The feasible set } \Omega \text { defined by } \\
& \quad \Omega=\left\{\left(S_{h}, E_{h}, I_{h}, R_{h}, S_{m}, E_{m}, I_{m}\right) \in \mathbb{R}_{+}^{7}: N_{h}(t) \leq N_{h 0} \text { and } N_{m}(t) \leq N_{m 0}\right\}
\end{aligned}
$$

is a compact set of $\mathbb{R}_{+}^{7}$ and $g(Y(t))=\left(g_{1}, g_{2}, g_{3}, g_{4}, g_{5}, g_{6}, g_{7}\right)$, where:

$$
\left\{\begin{array}{l}
g_{1}(Y(t))=\frac{\mathrm{d} S_{h}}{\mathrm{~d} t}=\mu N_{h}-\beta_{h}(v) \frac{I_{m}}{N_{h}} S_{h}-\mu S_{h}+\theta_{h} R_{h} \\
g_{2}(Y(t))=\frac{\mathrm{d} E_{h}}{\mathrm{~d} t}=\beta_{h}(v) \frac{I_{m}}{N_{h}} S_{h}-\left(\sigma_{h}+\mu\right) E_{h} \\
g_{3}(Y(t))=\frac{\mathrm{d} I_{h}}{\mathrm{~d} t}=\sigma_{h} E_{h}-\left(\mu+\delta+\gamma_{h}(v)\right) I_{h} \\
g_{4}(Y(t))=\frac{\mathrm{d} R_{h}}{\mathrm{~d} t}=\gamma_{h}(v) I_{h}-\left(\mu+\theta_{h}\right) R_{h} \\
g_{5}(Y(t))=\frac{\mathrm{d} S_{m}}{\mathrm{~d} t}=\mu_{m} N_{m}-\beta_{m} \frac{I_{h}}{N_{h}} S_{m}-\mu_{m} S_{m} \\
g_{6}(Y(t))=\frac{\mathrm{d} E_{m}}{\mathrm{~d} t}=\beta_{m} \frac{I_{h}}{N_{h}} S_{m}-\left(\sigma_{m}+\mu_{m}\right) E_{m} \\
g_{7}(Y(t))=\frac{\mathrm{d} I_{m}}{\mathrm{~d} t}=\sigma_{m} E_{m}-\left(\mu_{m}+\delta_{m}\right) I_{m}
\end{array}\right.
$$

Theorem 1. Under the initial condition given in (17), the system (10)-(16) 
admits a unique solution, non negative and bounded for all $t \geq 0$ in $\Omega=\left\{\left(S_{h}, E_{h}, I_{h}, R_{h}, S_{m}, E_{m}, I_{m}\right) \in \mathbb{R}_{+}^{7}: N_{h}(t) \leq N_{h 0}\right.$ and $\left.N_{m}(t) \leq N_{m 0}\right\}$.

Proof. All functions $g_{i}$ of system (21) are continuous functions on $\Omega$. Hence, the vector function $g$ is differentiable. This means that all partial derivatives exist and are continuous on $\Omega$. Therefore, by the existence and uniqueness theorem [30], there exists a unique solution of system (21) for the initial condition (17). Additionally, suppose that $Y(t)$ is a solution of system (10)-(16) for $Y(0) \geq 0$, and let $t_{0}$ be the smallest positive $t$ such that $S_{h}\left(t_{0}\right)=0$ or $E_{h}\left(t_{0}\right)=0$ or $I_{h}\left(t_{0}\right)=0$ or $R_{h}\left(t_{0}\right)=0$ or $S_{m}\left(t_{0}\right)=0$ or $E_{m}\left(t_{0}\right)=0$ or $I_{m}\left(t_{0}\right)=0$. By continuity of functions $S_{h}, E_{h}, I_{h}, R_{h}, S_{m}, E_{m}$ and $I_{m}$ there exits $t^{*}>t_{0}$ such that if $S_{h}\left(t_{0}\right)=0$, then from Equation (10), we have:

$\frac{\mathrm{d} S_{h}}{\mathrm{~d} t}\left(t_{0}\right)=\mu N_{h}\left(t_{0}\right)+\theta_{h} R_{h}\left(t_{0}\right) \geq 0$ for all $t \in\left[t_{0}, t^{*}\right]$. Thus, $S_{h}(t) \geq 0$ for all $t \in\left[t_{0}, t^{*}\right]$. Consequently, $S_{h}$ is non negative for all $t$. In the same way, we can establish the non negativity of $E_{h}, I_{h}, R_{h}, S_{m}, E_{m}$ and $I_{m}$ for all $t \geq 0$.

Therefore, the solutions $Y(t)$ of system (10)-(16) are non negatives for all $t \geq 0$ and that solutions remain positive for all the time. Consequently, the feasible set $\Omega$ is positively-invariant for all $t \geq 0$. Hence, the model (10)-(16) is well-posed and biologically meaningful. Finally, the solution $Y(t)$ of system (10)-(16) is bounded because the limit to infinity of the total humans population is less than the initial human population, that is

$$
\lim _{t \rightarrow+\infty} N_{h}(t) \leq N_{h 0},
$$

and the limit to infinity of the total mosquitoes population is less than the initial mosquitoes population, that is

$$
\lim _{t \rightarrow+\infty} N_{m}(t) \leq N_{m 0}
$$

\section{Analytical Analysis}

In this section, we introduce the fuzzy equilibrium of a fuzzy model. We introduce and compute the fuzzy basic reproduction number of fuzzy SEIRS-SEI model for malaria using the expected value of a fuzzy variable introduced by Baoding Liu and Y.-K. Liu [23] and finally we introduce the fuzzy global stabilities of the fuzzy SEIRS-SEI model for malaria.

\subsection{Fuzzy Equilibrium of the Model}

Theorem 2. Let given the system (10)-(16) with initial conditions (17) defined on the positively-invariant compact set $\Omega$. If $v \leq v_{\min }$, the system (10)-(16) admits only one equilibrium point noted and given by:

$$
Y^{0}=\left(S_{h}^{0}, E_{h}^{0}, I_{h}^{0}, R_{h}^{0}, S_{m}^{0}, E_{m}^{0}, I_{m}^{0}\right)=\left(N_{h}, 0,0,0, N_{m}, 0,0\right) ;
$$

and if $v>v_{\min }$, the system (10)-(16) admits at least two equilibrium points. The first one is given and noted by: 


$$
Y_{1}^{*}=\left(S_{1 h}^{*}, E_{1 h}^{*}, I_{1 h}^{*}, R_{1 h}^{*}, S_{1 m}^{*}, E_{1 m}^{*}, I_{1 m}^{*}\right),
$$

where $S_{1 h}^{*} \neq 0, E_{1 h}^{*} \neq 0, I_{1 h}^{*} \neq 0, R_{1 h}^{*} \neq 0, S_{1 m}^{*} \neq 0, E_{1 m}^{*} \neq 0$ and $I_{1 m}^{*} \neq 0$. The second one is given and noted by:

$$
Y_{2}^{*}=\left(S_{2 h}^{*}, E_{2 h}^{*}, I_{2 h}^{*}, R_{2 h}^{*}, S_{2 m}^{*}, E_{2 m}^{*}, I_{2 m}^{*}\right),
$$

where $S_{2 h}^{*} \neq 0, E_{2 h}^{*} \neq 0, I_{2 h}^{*} \neq 0, R_{2 h}^{*} \neq 0, S_{2 m}^{*} \neq 0, E_{2 m}^{*} \neq 0$ and $I_{2 m}^{*} \neq 0$.

Proof. The equilibrium of system (10)-(16) are obtained when $\frac{\mathrm{d} S_{h}}{\mathrm{~d} t}=\frac{\mathrm{d} E_{h}}{\mathrm{~d} t}=\frac{\mathrm{d} I_{h}}{\mathrm{~d} t}=\frac{\mathrm{d} R_{h}}{\mathrm{~d} t}=\frac{\mathrm{d} S_{m}}{\mathrm{~d} t}=\frac{\mathrm{d} E_{m}}{\mathrm{~d} t}=\frac{\mathrm{d} I_{m}}{\mathrm{~d} t}=0$. A point $\left(S_{h}, E_{h}, I_{h}, R_{h}, S_{m}, E_{m}, I_{m}\right)$ of $\Omega$ is an equilibrium point of system (10)-(16) if the system (22)-(28) below holds.

$$
\begin{gathered}
\mu N_{h}-\beta_{h}(v) \frac{I_{m}}{N_{h}} S_{h}-\mu S_{h}+\theta_{h} R_{h}=0 \\
\beta_{h}(v) \frac{I_{m}}{N_{h}} S_{h}-\left(\sigma_{h}+\mu\right) E_{h}=0 \\
\sigma_{h} E_{h}-\left(\mu+\delta+\gamma_{h}(v)\right) I_{h}=0 \\
\gamma_{h}(v) I_{h}-\left(\mu+\theta_{h}\right) R_{h}=0 \\
\mu_{m} N_{m}-\beta_{m} \frac{I_{h}}{N_{h}} S_{m}-\mu_{m} S_{m}=0 \\
\beta_{m} \frac{I_{h}}{N_{h}} S_{m}-\left(\sigma_{m}+\mu_{m}\right) E_{m}=0 \\
\sigma_{m} E_{m}-\left(\mu_{m}+\delta_{m}\right) I_{m}=0
\end{gathered}
$$

From Equation (25), we have:

$$
R_{h}=\frac{\gamma_{h}(v)}{\mu+\theta_{h}} I_{h} .
$$

From Equation (24), we obtain:

$$
E_{h}=\frac{\mu+\delta+\gamma_{h}(v)}{\sigma_{h}} I_{h} .
$$

Putting Equation (29) into Equation (22), we get:

$$
S_{h}=\frac{\mu N_{h}+\frac{\theta_{h} \gamma_{h}(v)}{\mu+\theta_{h}} I_{h}}{\mu+\beta_{h}(v) \frac{I_{m}}{N_{h}}} .
$$

From Equation (26), we have:

$$
S_{m}=\frac{\mu_{m} N_{m}}{\mu_{m}+\beta_{m} \frac{I_{h}}{N_{h}}} .
$$

From Equation (27), we get:

$$
E_{m}=\frac{\beta_{m} \frac{I_{h}}{N_{h}} S_{m}}{\mu_{m}+\sigma_{m}} .
$$


From Equation (28), we obtain:

$$
I_{m}=\frac{\sigma_{m} E_{m}}{\mu_{m}+\delta_{m}}
$$

The substitution of Equation (32) into (33) gives:

$$
E_{m}=\frac{\beta_{m} \mu_{m} N_{m} I_{h}}{\left(\mu_{m} N_{h}+\beta_{m} I_{h}\right)\left(\mu_{m}+\sigma_{m}\right)} .
$$

The substitution of Equation (35) into (34) gives:

$$
I_{m}=\frac{\beta_{m} \sigma_{m} \mu_{m} N_{m} I_{h}}{\left(\mu_{m} N_{h}+\beta_{m} I_{h}\right)\left(\mu_{m}+\sigma_{m}\right)\left(\mu_{m}+\delta_{m}\right)} .
$$

Putting Equation (36) into Equation (31) gives:

$$
S_{h}=\frac{\mu N_{h}+\frac{\theta_{h} \gamma_{h}(v)}{\mu+\theta_{h}} I_{h}}{\mu+\beta_{h}(v) \frac{\beta_{m} \sigma_{m} \mu_{m} N_{m} I_{h}}{N_{h}\left(\mu_{m} N_{h}+\beta_{m} I_{h}\right)\left(\mu_{m}+\sigma_{m}\right)\left(\mu_{m}+\delta_{m}\right)}} .
$$

The equilibrium points are expressed as implicit functions in terms of infectious humans $I_{h}$, where:

$$
\begin{aligned}
I_{h}= & N_{h}-\left(S_{h}+E_{h}+R_{h}\right) \\
= & N_{h}-\left[\frac{\mu N_{h}+\frac{\theta_{h} \gamma_{h}(v)}{\mu+\theta_{h}} I_{h}}{\mu+\beta_{h}(v) \frac{\beta_{m} \sigma_{m} \mu_{m} N_{m} I_{h}}{N_{h}\left(\mu_{m} N_{h}+\beta_{m} I_{h}\right)\left(\mu_{m}+\sigma_{m}\right)\left(\mu_{m}+\delta_{m}\right)}}\right. \\
& \left.+\frac{\mu+\delta+\gamma_{h}(v)}{\sigma_{h}} I_{h}+\frac{\gamma_{h}(v)}{\mu+\theta_{h}} I_{h}\right] .
\end{aligned}
$$

Case 1: If $v \leq v_{\min }$. In this case $\beta_{h}(v)=0$ and $\gamma_{h}(v)>0$ Substitute it into Equation (38), we obtain:

$$
I_{h}=0
$$

Substituting Equation (39) into Equations (29), (30), (32), (35), (36) and (37), we obtain respectively: $R_{h}=0, E_{h}=0, S_{m}=N_{m}, E_{m}=0, I_{m}=0$ and $S_{h}=N_{h}$. Therefore, we obtain:

$$
Y^{0}=\left(S_{h}^{0}, E_{h}^{0}, I_{h}^{0}, R_{h}^{0}, S_{m}^{0}, E_{m}^{0}, I_{m}^{0}\right)=\left(N_{h}, 0,0,0, N_{m}, 0,0\right),
$$

the only one equilibrium point when $v \leq v_{\min }$, called "Disease-free equilibrium point (DFE)". This situation occurs when there is no malaria in the population. The biological implication of this is, when the amount of parasite is less than a minimum amount required in the body for disease transmission, malaria will die out.

Case 2: If $v_{\min }<v<v_{M}$. From here, $\beta_{h}(v)=\frac{v-v_{\min }}{v_{M}-v_{\min }}>0$ and $\gamma_{h}(v)>0$. 
In this case, the equilibrium points are given by:

$$
Y_{1}^{*}=\left(S_{1 h}^{*}, E_{1 h}^{*}, I_{1 h}^{*}, R_{1 h}^{*}, S_{1 m}^{*}, E_{1 m}^{*}, I_{1 m}^{*}\right),
$$

where the vector's components are given by the implicit-solutions functions (37), (30), (38), (29), (32), (35) and (36) respectively.

Case 3: If $v_{M} \leq v \leq v_{\max }$. In this case, $\beta_{h}(v)=1$ and $\gamma_{h}(v)>0$. In this case, Equations (37) and (38) become:

$$
S_{h}=\frac{\mu N_{h}+\frac{\theta_{h} \gamma_{h}(v)}{\mu+\theta_{h}} I_{h}}{\mu+\frac{\beta_{m} \sigma_{m} \mu_{m} N_{m} I_{h}}{N_{h}\left(\mu_{m} N_{h}+\beta_{m} I_{h}\right)\left(\mu_{m}+\sigma_{m}\right)\left(\mu_{m}+\delta_{m}\right)}},
$$

and

$$
\begin{aligned}
I_{h}= & N_{h}-\left[\frac{\mu N_{h}+\frac{\theta_{h} \gamma_{h}(v)}{\mu+\theta_{h}} I_{h}}{\mu+\frac{\beta_{m} \sigma_{m} \mu_{m} N_{m} I_{h}}{N_{h}\left(\mu_{m} N_{h}+\beta_{m} I_{h}\right)\left(\mu_{m}+\sigma_{m}\right)\left(\mu_{m}+\delta_{m}\right)}}\right. \\
& \left.+\frac{\mu+\delta+\gamma_{h}(v)}{\sigma_{h}} I_{h}+\frac{\gamma_{h}(v)}{\mu+\theta_{h}} I_{h}\right] .
\end{aligned}
$$

We obtain the equilibrium point

$$
Y_{2}^{*}=\left(S_{2 h}^{*}, E_{2 h}^{*}, I_{2 h}^{*}, R_{2 h}^{*}, S_{2 m}^{*}, E_{2 m}^{*}, I_{2 m}^{*}\right),
$$

where the vector's components are given by the implicit-solutions functions (41), (30), (42), (29), (32), (35) and (36) respectively.

The equilibrium points obtained in cases 2 and 3 are called "endemic equilibriums", they occur when malaria persists in the population. Thus, when the amount of parasites is greater than the minimum amount required, malaria occurs and persists in the population.

\subsection{Fuzzy Basic Reproduction Number $\mathcal{R}_{0}^{f}$}

The classical basic reproduction number, noted by $\mathcal{R}_{0}$, is defined as the average number of secondary malaria cases generated by a single infected individual introduced into a healthy population during its infectious period. In the classical analysis, if $\mathcal{R}_{0}>1$ the malaria continues to propagate in the population and becomes endemic. If $\mathcal{R}_{0}<1$ the malaria disappears in the population. Using the classical Next Generation Matrix as explained in [31], we have four disease compartments $\left(E_{h}, I_{h}, E_{m}\right.$ and $\left.I_{m}\right)$ and three non-disease compartments $\left(S_{h}, R_{h}\right.$ and $\left.S_{m}\right)$. Mathematically, the basic reproduction number $\mathcal{R}_{0}$ is defined as the spectral radius of the next generation matrix $F V^{-1}$, where $F$ is the matrix of transmission terms and $V$ is the matrix of transition terms between the model state. For system (10)-(16), matrices $F$ and $V$ are given respectively by: 


$$
F=\left(\begin{array}{cccc}
0 & 0 & 0 & \beta_{h}(v) \\
0 & 0 & 0 & 0 \\
0 & \beta_{m} \frac{S_{m}}{N_{h}} & 0 & 0 \\
0 & 0 & 0 & 0
\end{array}\right) \text { and } V=\left(\begin{array}{cccc}
k_{1} & 0 & 0 & 0 \\
-\sigma_{h} & k_{2} & 0 & 0 \\
0 & 0 & k_{3} & 0 \\
0 & 0 & -\sigma_{m} & k_{4}
\end{array}\right) \text {, }
$$

where $k_{1}=\mu+\sigma_{h}, k_{2}=\mu+\delta+\gamma_{h}(v), k_{3}=\mu_{m}+\sigma_{m} \quad$ and $\quad k_{4}=\mu_{m}+\delta_{m}$. The simple computations give:

$$
V^{-1}=\left(\begin{array}{cccc}
\frac{1}{k_{1}} & 0 & 0 & 0 \\
\frac{\sigma_{h}}{k_{1} k_{2}} & \frac{1}{k_{2}} & 0 & 0 \\
0 & 0 & \frac{1}{k_{3}} & 0 \\
0 & 0 & \frac{\sigma_{m}}{k_{3} k_{4}} & \frac{1}{k_{4}}
\end{array}\right), V^{-1} F=\left(\begin{array}{cccc}
0 & 0 & 0 & \frac{\beta_{h}(v)}{k_{1}} \\
0 & 0 & 0 & \frac{\beta_{h}(v) \sigma_{h}}{k_{1} k_{2}} \\
0 & \frac{\beta_{m} N_{m}}{k_{3} N_{h}} & 0 & 0 \\
0 & \frac{\beta_{m} \sigma_{m} N_{m}}{k_{3} k_{4} N_{h}} & 0 & 0
\end{array}\right)
$$

and

$$
F V^{-1}=\left(\begin{array}{cccc}
0 & 0 & \frac{\beta_{h}(v) \sigma_{m}}{k_{3} k_{4}} & \frac{\beta_{h}(v)}{k_{4}} \\
0 & 0 & 0 & 0 \\
\frac{N_{m} \beta_{m} \sigma_{h}}{N_{h} k_{1} k_{2}} & \frac{N_{m} \beta_{m}}{N_{h} k_{2}} & 0 & 0 \\
0 & 0 & 0 & 0
\end{array}\right) .
$$

The basic reproduction number is given by:

$$
\begin{aligned}
\mathcal{R}_{0}(v) & =\rho\left(F V^{-1}\right) \\
& =\sqrt{\frac{\beta_{h}(v) \beta_{m} \sigma_{h} \sigma_{m}}{k_{1} k_{2} k_{3} k_{4}} \frac{N_{m}}{N_{h}} .}
\end{aligned}
$$

As computed, $\mathcal{R}_{0}$ is a function of the amount of parasites (parasitic viral load) $v$. We analyze it for different amount of parasites.

Case 1: If $v \leq v_{\min }$. In this case $\beta_{h}(v)=0$ and $\gamma_{h}(v)>0$ which implies that $k_{2}>0$. In this case, we obtain $\mathcal{R}_{0}(v)=0$, which coincides with the case where there is no malaria in the population. The disease dies out.

Case 2: If $v_{\min }<v<v_{M}$. In this case, $\beta_{h}(v)=\frac{v-v_{\min }}{v_{M}-v_{\min }}>0$ and $\gamma_{h}(v)>0$ which implies $k_{2}>0$. In this case, we have:

$$
0<\mathcal{R}_{0}(v) \equiv \sqrt{\frac{N_{m}}{N_{h}} \frac{\left(v-v_{\min }\right) \beta_{m} \sigma_{h} \sigma_{m}}{\left(v_{M}-v_{\min }\right) k_{1} k_{2} k_{3} k_{4}} .}
$$

Case 3: If $v_{M} \leq v \leq v_{\max }$. In this case $\beta_{h}(v)=1$ and $\gamma_{h}(v)>0$ which implies that $k_{2}>0$. In this case, we have:

$$
0<\mathcal{R}_{0}(v) \leq \sqrt{\frac{N_{m}}{N_{h}} \frac{\beta_{m} \sigma_{h} \sigma_{m}}{k_{1} k_{2} k_{3} k_{4}}} .
$$


The basic reproduction number $\mathcal{R}_{0}(v)$ is an increasing function of the parasitic viral load $v$. Additionally, $\mathcal{R}_{0}(v)$ is a bounded function with the bound $\sqrt{\frac{N_{m}}{N_{h}} \frac{\beta_{m} \sigma_{h} \sigma_{m}}{k_{1} k_{2} k_{3} k_{4}}}>0$. The basic reproduction number $\mathcal{R}_{0}(v)$ is well-defined as a fuzzy variable. Consequently the expected value of $\mathcal{R}_{0}(v)$ denoted by $E\left[\mathcal{R}_{0}(v)\right]$, is well-defined. The fuzzy number $\mathcal{R}_{0}(v)$ can be expressed as:

$$
\mathcal{R}_{0}(v)=\left(0, \sqrt{\frac{N_{m}}{N_{h}} \frac{\left(v-v_{\text {min }}\right) \beta_{m} \sigma_{h} \sigma_{m}}{\left(v_{M}-v_{\text {min }}\right) k_{1} k_{2} k_{3} k_{4}}}, \sqrt{\frac{N_{m}}{N_{h}} \frac{\beta_{m} \sigma_{h} \sigma_{m}}{k_{1} k_{2} k_{3} k_{4}}}\right),
$$

which is a triangular fuzzy number. Referring to Formula (6), we define therefore the fuzzy basic reproduction number $\mathcal{R}_{0}^{f}$ for a triangular fuzzy number $\mathcal{R}_{0}(v)$ as in Proposition 1 below:

Proposition 1 (Fuzzy basic reproduction number, $\mathcal{R}_{0}^{f}$ ) The fuzzy basic reproduction number $\mathcal{R}_{0}^{f}$ of the malaria SEIRS-SEI model is given by.

$$
\begin{aligned}
\mathcal{R}_{0}^{f} & =E\left[\mathcal{R}_{0}(v)\right] \\
& =\frac{1}{4}\left(2 \sqrt{\frac{v-v_{\min }}{v_{M}-v_{\min }}}+1\right) \sqrt{\frac{N_{m} \beta_{m} \sigma_{h} \sigma_{m}}{N_{h} k_{1} k_{2} k_{3} k_{4}}} \text {, for } v>v_{\text {min }} ;
\end{aligned}
$$

where $E[$.$] denotes the expected value of a fuzzy number defined in Formula$ (6).

\subsection{Fuzzy Global Stability of the Disease-Free Equilibrium}

Theorem 3. The disease-free equilibrium $Y^{0}=\left(N_{h}, 0,0,0, N_{m}, 0,0\right)$ of system (10)-(16) is globally asymptotically stable in the positively-invariant compact set $\Omega$ if $v \leq v_{\min }$ or $\mathcal{R}_{0}(v) \leq 1$. If $\mathcal{R}_{0}(v)>1$ and if $v>v_{\text {min }}$, the disease-free equilibrium is unstable, the system is uniformly persistent and there is a co-existing equilibrium point in the interior of $\Omega$.

Proof. We use the classical Lyapunov function theory to derive the fuzzy global asymptotic stability of the disease-free equilibrium. To construct the Lyapunov function, we use the classical matrix-theoretical method as explained in [24]. Assume that $x=\left(E_{h}, I_{h}, E_{m}, I_{m}\right)^{\mathrm{T}}$ and $y=\left(S_{h}, R_{h}, S_{m}\right)^{\mathrm{T}}$. The computations of $f(x, y)$ give:

$$
\begin{aligned}
f(x, y) & =(F-V) x-\mathcal{F}(x, y)+\mathcal{V}(x, y) \\
& =\left(\begin{array}{c}
\beta_{h}(v) I_{m}\left(1-\frac{S_{h}}{N_{h}}\right) \\
0 \\
\beta_{m}\left(N_{m}-S_{m}\right) \frac{I_{h}}{N_{h}} \\
0
\end{array}\right)
\end{aligned}
$$

and 


$$
V^{-1} F=\left(\begin{array}{cccc}
0 & 0 & 0 & \frac{\beta_{h}(v)}{k_{1}} \\
0 & 0 & 0 & \frac{\beta_{h}(v) \sigma_{h}}{k_{1} k_{2}} \\
0 & \frac{\beta_{m} N_{m}}{k_{3} N_{h}} & 0 & 0 \\
0 & \frac{\beta_{m} \sigma_{m} N_{m}}{N_{h} k_{3} k_{4}} & 0 & 0
\end{array}\right) .
$$

Since $S_{m} \leq N_{m}$ and $S_{h} \leq N_{h}, f(x, y) \geq 0$. In addition, $F \geq 0, V^{-1} \geq 0$ and $f\left(x,\left(N_{h}, 0, N_{m}\right)^{\mathrm{T}}\right)=0$ in the feasible set $\Omega$. Therefore, since the matrix $V^{-1} F$ is reducible, we use Theorem 2.1 of [24] to construct the Lyapunov function of system (10)-(16). Let $w^{\mathrm{T}}=\left(w_{1}, w_{2}, w_{3}, w_{4}\right) \geq 0$ be the left eigenvector of the non-negative matrix $V^{-1} F$ corresponding to the eigenvalue $\mathcal{R}_{0}(v)$. Then:

$$
\left(w_{1}, w_{2}, w_{3}, w_{4}\right) V^{-1} F=\left(w_{1}, w_{2}, w_{3}, w_{4}\right) \mathcal{R}_{0}(v) .
$$

From the left hand side of (46), we have:

$$
\begin{aligned}
& \left(w_{1}, w_{2}, w_{3}, w_{4}\right) V^{-1} F=\left(w_{1}, w_{2}, w_{3}, w_{4}\right)\left(\begin{array}{cccc}
0 & 0 & 0 & \frac{\beta_{h}(v)}{k_{1}} \\
0 & 0 & 0 & \frac{\beta_{h}(v) \sigma_{h}}{k_{1} k_{2}} \\
0 & \frac{\beta_{m} N_{m}}{k_{3} N_{h}} & 0 & 0 \\
0 & \frac{\beta_{m} \sigma_{m} N_{m}}{N_{h} k_{3} k_{4}} & 0 & 0
\end{array}\right) \\
& =\left(0, \frac{\beta_{m} N_{m}}{k_{3} N_{h}} w_{3}+\frac{N_{m} \beta_{m} \sigma_{m}}{N_{h} k_{3} k_{4}} w_{4}, 0, \frac{\beta_{h}(v)}{k_{1}} w_{1}+\frac{\beta_{h}(v) \sigma_{h}}{k_{1} k_{2}} w_{2}\right) .
\end{aligned}
$$

Equating Equations (47) and (46), we obtain: $w_{1}=w_{3}=0$ and $w_{4}=\frac{\left(\beta_{h}(v) \sigma_{h}+k_{1} k_{2} \mathcal{R}_{0}(v)\right) N_{h} k_{3} k_{4}}{k_{1} k_{2}\left(N_{m} \beta_{m} \sigma_{m}+N_{h} k_{3} k_{4} \mathcal{R}_{0}(v)\right)} w_{2}$, with $w_{2} \in \mathbb{R}^{+}$. Thus $w^{\mathrm{T}}=\left(0, w_{2}, 0, \frac{\left(\beta_{h}(v) \sigma_{h}+k_{1} k_{2} \mathcal{R}_{0}(v)\right) N_{h} k_{3} k_{4}}{k_{1} k_{2}\left(N_{m} \beta_{m} \sigma_{m}+N_{h} k_{3} k_{4} \mathcal{R}_{0}(v)\right)} w_{2}\right)$. Therefore, by Theorem 2.1 of [24], the Lyapunov function $L$ is constructed as follows:

$$
\begin{aligned}
& L=w^{\mathrm{T}} V^{-1} x \\
& =\left(0, w_{2}, 0, \frac{\left(\beta_{h}(v) \sigma_{h}+k_{1} k_{2} \mathcal{R}_{0}(v)\right) N_{h} k_{3} k_{4}}{k_{1} k_{2}\left(N_{m} \beta_{m} \sigma_{m}+N_{h} k_{3} k_{4} \mathcal{R}_{0}(v)\right)} w_{2}\right)\left(\begin{array}{cccc}
\frac{1}{k_{1}} & 0 & 0 & 0 \\
\frac{\sigma_{h}}{k_{1} k_{2}} & \frac{1}{k_{2}} & 0 & 0 \\
0 & 0 & \frac{1}{k_{3}} & 0 \\
0 & 0 & \frac{\sigma_{m}}{k_{3} k_{4}} & \frac{1}{k_{4}}
\end{array}\right)\left(\begin{array}{c}
E_{h} \\
I_{h} \\
E_{m} \\
I_{m}
\end{array}\right)
\end{aligned}
$$




$$
\begin{aligned}
= & \left(\frac{\sigma_{h}}{k_{1} k_{2}} E_{h}+\frac{1}{k_{2}} I_{h}+\frac{\sigma_{m} N_{h}\left(\beta_{h}(v) \sigma_{h}+k_{1} k_{2} \mathcal{R}_{0}(v)\right)}{k_{1} k_{2}\left(N_{m} \beta_{m} \sigma_{m}+k_{3} k_{4} N_{h} \mathcal{R}_{0}(v)\right)} E_{m}\right. \\
& \left.+\frac{k_{3} N_{h}\left(\beta_{h}(v) \sigma_{h}+k_{1} k_{2} \mathcal{R}_{0}(v)\right)}{k_{1} k_{2}\left(N_{m} \beta_{m} \sigma_{m}+k_{3} k_{4} N_{h} \mathcal{R}_{0}(v)\right)} I_{m}\right) w_{2}>0, \forall Y \neq Y^{0} ;
\end{aligned}
$$

is a Lyapunov function for the system (10)-(16). The differentiation of $L$ with respect to $t$ gives:

$$
\begin{aligned}
\dot{L}= & w^{\mathrm{T}} V^{-1} \dot{x}=w^{\mathrm{T}} V^{-1}(F-V) x-w^{\mathrm{T}} V^{-1} f(x, y) \\
= & \left(\mathcal{R}_{0}(v)-1\right) w^{\mathrm{T}} x-w^{\mathrm{T}} V^{-1} f(x, y) \\
= & \left(\mathcal{R}_{0}(v)-1\right)\left(w_{2} I_{h}+\frac{k_{3} k_{4} N_{h}\left(\beta_{h}(v) \sigma_{h}+k_{1} k_{2} \mathcal{R}_{0}(v)\right) w_{2}}{k_{1} k_{2}\left(N_{m} \beta_{m} \sigma_{m}+k_{3} k_{4} N_{h} \mathcal{R}_{0}(v)\right)} I_{m}\right) \\
& -\frac{\beta_{h}(v) \sigma_{h} w_{2}}{k_{1} k_{2}}\left(1-\frac{S_{h}}{N_{h}}\right) I_{m}-\frac{\beta_{m} \sigma_{m}\left(\beta_{h}(v) \sigma_{h}+k_{1} k_{2} \mathcal{R}_{0}(v)\right) w_{2}}{k_{1} k_{2}\left(N_{m} \beta_{m} \sigma_{m}+k_{3} k_{4} N_{h} \mathcal{R}_{0}(v)\right)}\left(N_{m}-S_{m}\right) I_{h} .
\end{aligned}
$$

Since $S_{h} \leq N_{h}$ and $S_{m} \leq N_{m}$, if $v \leq v_{\text {min }}$, then $\beta_{h}(v)=0$ and $\mathcal{R}_{0}(v)=0$ which implies that $\dot{L}<0$. Additionally, $\dot{L}<0$ if $\mathcal{R}_{0}(v) \leq 1$. Furthermore, $\dot{L}=0$ implies that $I_{h}=I_{m}=0, S_{h}=N_{h}$ and $S_{m}=N_{m}$. Thus, the largest invariant set of the model when $\dot{L}=0$ in the interior of $\Omega$ is the singleton $\left\{\left(N_{h}, 0,0,0, N_{m}, 0,0\right)\right\}$. Therefore, by the LaSalle's invariance principle [32], the disease-free equilibrium $Y^{0}=\left(N_{h}, 0,0,0, N_{m}, 0,0\right)$ is globally asymptotically stable provided that $\mathcal{R}_{0}(v) \leq 1$ or $v \leq v_{\text {min }}$. In addition, for $\mathcal{R}_{0}(v)>1$ and $v>v_{\min }$, then $\dot{L}>0$ in $\Omega$ if $S_{h}=N_{h}$ and $S_{m}=N_{m}$. Therefore, by continuity, $\dot{L}$ remains positive in a small neighbourhood of the disease-free equilibrium $\left\{\left(N_{h}, 0,0,0, N_{m}, 0,0\right)\right\}$, which implies that $\left\{\left(N_{h}, 0,0,0, N_{m}, 0,0\right)\right\}$ is unstable and using Theorem 2.2 of [24], the system (10)-(16) is uniformly persistent, implies that there exists at least one endemic point, noted and defined by (40) or (43) in the interior of $\Omega$.

Remark 1. The biological implication of the above Theorem is malaria can diet out from the population if the amount of parasites is less that the minimum amount required for malaria transmission $\left(v \leq v_{\min }\right)$ or if the basic reproduction number is less than or equal to the unity $\left(\mathcal{R}_{0}(v) \leq 1\right)$. If $\mathcal{R}_{0}(v)>1$ and if the amount of parasites is greater than the minimum amount required for the malaria transmission $\left(v>v_{\min }\right)$, malaria persists uniformly in the population and becomes endemic.

\subsection{Fuzzy Global Stability of the Endemic Equilibrium}

Theorem 4. The endemic equilibrium $Y^{*}=\left(S_{h}^{*}, E_{h}^{*}, I_{h}^{*}, R_{h}^{*}, S_{m}^{*}, E_{m}^{*}, I_{m}^{*}\right) \in \operatorname{int}(\Omega)$ of system (10)-(16) is globally asymptotically stable in the interior of the positively-invariant compact set $\Omega$ if $v>v_{\min }$ (i.e. $v_{\min }<v \leq v_{M}$ or $\left.v_{M}<v \leq v_{\max }\right)$ and $\mathcal{R}_{0}(v)>1$.

Proof. As used in Theorem (3), we use the classical Lyapunov function theory to derive this fuzzy global asymptotic stability. We use the classical graph-theoretical method introduced by Zhisheng Shuai and Pauline van den Driessche [24]. For 
the construction of a Lyapunov function, let define:

$$
L_{i}: \Omega \rightarrow \mathbb{R}, i \in\{1,2,3,4,5,6,7\}
$$

where:

$$
\begin{array}{ll}
L_{1}=S_{h}-S_{h}^{*}-S_{h}^{*} \ln \left(S_{h} / S_{h}^{*}\right) ; & L_{2}=E_{h}-E_{h}^{*}-E_{h}^{*} \ln \left(E_{h} / E_{h}^{*}\right) \\
L_{3}=I_{h}-I_{h}^{*}-I_{h}^{*} \ln \left(I_{h} / I_{h}^{*}\right) ; & L_{4}=R_{h}-R_{h}^{*}-R_{h}^{*} \ln \left(R_{h} / R_{h}^{*}\right) \\
L_{5}=S_{m}-S_{m}^{*}-S_{m}^{*} \ln \left(S_{m} / S_{m}^{*}\right) ; & L_{6}=E_{m}-E_{m}^{*}-E_{m}^{*} \ln \left(E_{m} / E_{m}^{*}\right) \\
L_{7}=I_{m}-I_{m}^{*}-I_{m}^{*} \ln \left(I_{m} / I_{m}^{*}\right) . &
\end{array}
$$

The substitution of $Y^{*}=\left(S_{h}^{*}, E_{h}^{*}, I_{h}^{*}, R_{h}^{*}, S_{m}^{*}, E_{m}^{*}, I_{m}^{*}\right)$ into system (22)-(28) gives equalities: $\quad \mu N_{h}=\beta_{h}(v) \frac{I_{m}^{*}}{N_{h}} S_{h}^{*}+\mu S_{h}^{*}-\theta_{h} R_{h}^{*} ; \quad \sigma_{h}+\mu=\beta_{h}(v) \frac{I_{m}^{*}}{N_{h}} \frac{S_{h}^{*}}{E_{h}^{*}} ;$ $\mu+\delta+\gamma_{h}(v)=\sigma_{h} E_{h}^{*} / I_{h}^{*} ; \mu+\theta_{h}=\gamma_{h}(v) I_{h}^{*} / R_{h}^{*} ; \quad \mu_{m} N_{m}=\beta_{m} \frac{I_{h}^{*}}{N_{h}} S_{m}^{*}+\mu_{m} S_{m}^{*} ;$ $\sigma_{m}+\mu_{m}=\beta_{m} \frac{I_{h}^{*}}{N_{h}} \frac{S_{m}^{*}}{E_{m}^{*}}$ and $\mu_{m}+\delta_{m}=\sigma_{m} E_{m}^{*} / I_{m}^{*}$. We use these equalities and inequalities $1-x+\ln x \leq 0$ and $2-x-\frac{1}{x} \leq 0$ for $x>0$ in the differentiation of $L_{i}, i \in\{1,2,3,4,5,6,7\}$ with respect to $t$.

$$
\begin{aligned}
& \dot{L}_{1}=\left(1-\frac{S_{h}^{*}}{S_{h}}\right) \dot{S}_{h}=\left(1-\frac{S_{h}^{*}}{S_{h}}\right)\left(\mu N_{h}-\beta_{h}(v) \frac{I_{m}}{N_{h}} S_{h}-\mu S_{h}+\theta_{h} R_{h}\right) \\
& \leq \beta_{h}(v) \frac{I_{m}^{*} S_{h}^{*}}{N_{h}}\left(\frac{I_{m}}{I_{m}^{*}}-\frac{S_{h}^{*}}{S_{h}}-\ln \frac{I_{m}}{I_{m}^{*}}+\ln \frac{S_{h}^{*}}{S_{h}}\right)+\theta_{h} R_{h}^{*}\left(1-\frac{S_{h}^{*} R_{h}}{S_{h} R_{h}^{*}}+\frac{S_{h}^{*}}{S_{h}}+\frac{R_{h}}{R_{h}^{*}}\right) \\
& \leq \beta_{h}(v) \frac{I_{m}^{*} S_{h}^{*}}{N_{h}}\left(\frac{I_{m}}{I_{m}^{*}}-\frac{S_{h}^{*}}{S_{h}}-\ln \frac{I_{m}}{I_{m}^{*}}+\ln \frac{S_{h}^{*}}{S_{h}}\right)+\theta_{h} R_{h}^{*}\left(\frac{S_{h}^{*}}{S_{h}}+\frac{R_{h}}{R_{h}^{*}}-\ln \frac{S_{h}^{*}}{S_{h}}-\ln \frac{R_{h}}{R_{h}^{*}}\right) \\
&=a_{17} G_{17}+a_{14} G_{14} . \\
& \dot{L}_{2}=\left(1-\frac{E_{h}^{*}}{E_{h}}\right) \dot{E}_{h}=\left(1-\frac{E_{h}^{*}}{E_{h}}\right)\left(\beta_{h}(v) \frac{I_{m}}{N_{h}} S_{h}-\left(\sigma_{h}+\mu\right) E_{h}\right) \\
& \leq \beta_{h}(v) \frac{I_{m}^{*} S_{h}^{*}}{N_{h}}\left(\frac{S_{h} I_{m}}{S_{h}^{*} I_{m}^{*}}-\frac{E_{h}}{E_{h}^{*}}-\ln \frac{S_{h} I_{m}}{S_{h}^{*} I_{m}^{*}}-\ln \frac{E_{h}^{*}}{E_{h}}\right)=a_{21} G_{21} . \\
& \dot{L}_{3}=\left(1-\frac{I_{h}^{*}}{I_{h}}\right) \dot{I}_{h}=\left(1-\frac{I_{h}^{*}}{I_{h}}\right)\left(\sigma_{h} E_{h}-\left(\mu+\delta+\gamma_{h}(v)\right) I_{h}\right) \\
& \leq \sigma_{h} E_{h}^{*}\left(\frac{E_{h}}{E_{h}^{*}}-\frac{I_{h}}{I_{h}^{*}}-\ln \frac{E_{h}}{E_{h}^{*}}+\ln \frac{I_{h}}{I_{h}^{*}}\right)=a_{32} G_{32} . \\
& \dot{L}_{4}=\left(1-\frac{R_{h}^{*}}{R_{h}}\right) \dot{R}_{h}=\left(1-\frac{R_{h}^{*}}{R_{h}}\right)\left(\gamma_{h}(v) I_{h}-\left(\mu+\theta_{h}\right) R_{h}\right) \\
& \leq \gamma_{h}(v) I_{h}^{*}\left(\frac{I_{h}}{I_{h}^{*}}-\frac{R_{h}}{R_{h}^{*}}+\ln \frac{R_{h}}{R_{h}^{*}}-\ln \frac{I_{h}}{I_{h}^{*}}\right)=a_{43} G_{43} .
\end{aligned}
$$




$$
\begin{gathered}
\dot{L}_{5}=\left(1-\frac{S_{m}^{*}}{S_{m}}\right) \dot{S}_{m}=\left(1-\frac{S_{m}^{*}}{S_{m}}\right)\left(\mu_{m} N_{m}-\beta_{m} \frac{I_{h}}{N_{h}} S_{m}-\mu_{m} S_{m}\right) \\
\leq \beta_{m} \frac{I_{h}^{*} S_{m}^{*}}{N_{h}}\left(\frac{I_{h}}{I_{h}^{*}}-\frac{S_{m}^{*}}{S_{m}}-\ln \frac{I_{h}}{I_{h}^{*}}-\ln \frac{S_{m}}{S_{m}^{*}}\right)=a_{53} G_{53} . \\
\dot{L}_{6}=\left(1-\frac{E_{m}^{*}}{E_{m}}\right) \dot{E}_{m}=\left(1-\frac{E_{m}^{*}}{E_{m}}\right)\left(\beta_{m} \frac{I_{h}}{N_{h}} S_{m}-\left(\sigma_{m}+\mu_{m}\right) E_{m}\right) \\
\leq \beta_{m} \frac{I_{h}^{*} S_{m}^{*}}{N_{h}}\left(\frac{S_{m} I_{h}}{S_{m}^{*} I_{h}^{*}}-\frac{E_{m}}{E_{m}^{*}}-\ln \frac{S_{m} I_{h}}{S_{m}^{*} I_{h}^{*}}-\ln \frac{E_{m}^{*}}{E_{m}}\right)=a_{61} G_{61} . \\
\dot{L}_{7}=\left(1-\frac{I_{m}^{*}}{I_{m}}\right) \dot{I}_{m}=\left(1-\frac{I_{m}^{*}}{I_{m}}\right)\left(\sigma_{m} E_{m}-\left(\mu_{m}+\delta\right) I_{m}\right) \\
\leq \sigma_{m} E_{m}^{*}\left(\frac{E_{m}}{E_{m}^{*}}-\frac{I_{m}}{I_{m}^{*}}+\ln \frac{I_{m}}{I_{m}^{*}}-\ln \frac{E_{m}}{E_{m}^{*}}\right)=a_{76} G_{76} .
\end{gathered}
$$

Case 1: If $v \leq v_{\min }$, we have $\beta_{h}(v)=0$ and $\gamma_{h}(v)>0$. In this case, inequalities (48) and (49) become:

$$
\dot{L}_{1} \leq \theta_{h} R_{h}^{*}\left(\frac{S_{h}^{*}}{S_{h}}+\frac{R_{h}}{R_{h}^{*}}-\ln \frac{S_{h}^{*}}{S_{h}}-\ln \frac{R_{h}}{R_{h}^{*}}\right)=a_{14} G_{14} \text { and } \dot{L}_{2} \leq 0,
$$

respectively. Therefore, the weighted associated digraph $(G, A)$ is shown on Figure 4.

From this digraph, the first condition of Theorem 3.5 of [24] is satisfied, but no its second one. Thus, we cannot formulate a Lyapunov function of system (10)-(16) in this case.

Case 2: If $v>v_{\min },\left(v_{\min }<v \leq v_{M}\right.$ or $\left.v_{M}<v \leq v_{\max }\right)$, we have $\beta_{h}(v)>0$ and $\gamma_{h}(v)>0$. In this case, the weighted associated digraph $(G, A)$ is shown on Figure 5.

The weights of the digraph are: $a_{21}=a_{17}=\beta_{h}(v) \frac{I_{m}^{*} S_{h}^{*}}{N_{h}}, a_{14}=\theta_{h} R_{h}^{*}$, $a_{32}=\sigma_{h} E_{h}^{*}, a_{43}=\gamma_{h}(v) I_{h}^{*}, a_{53}=a_{61}=\beta_{m} \frac{I_{h}^{*} S_{m}^{*}}{N_{h}}$ and $a_{76}=\sigma_{m} E_{m}^{*}$, and all other

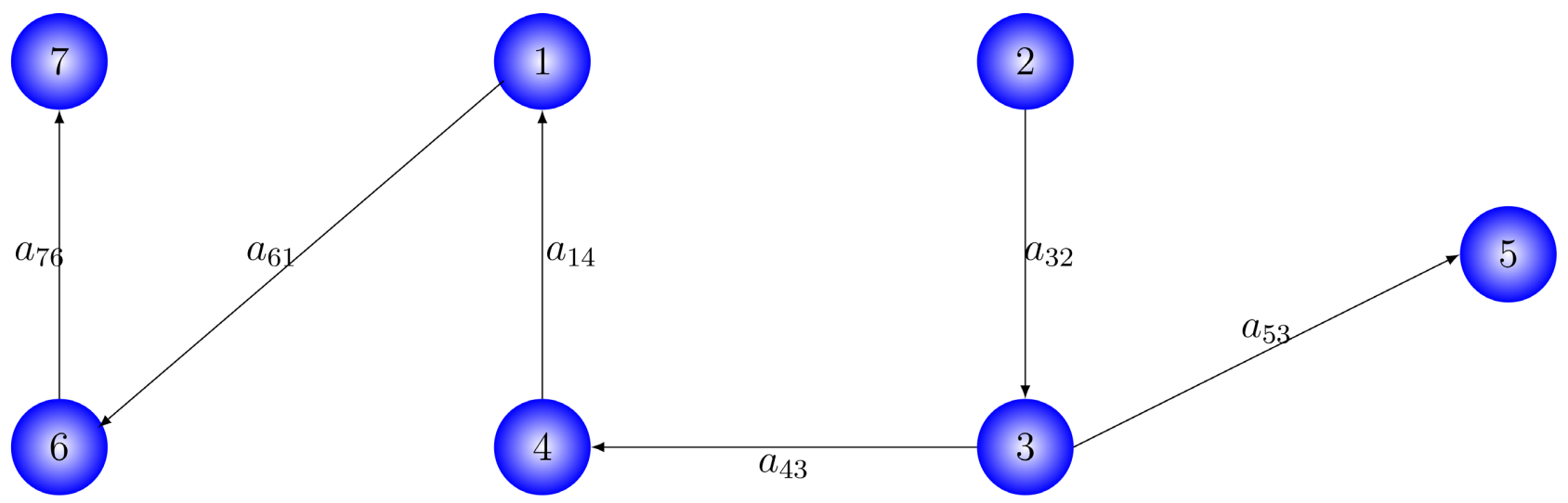

Figure 4. The weighted associated digraph $(G, A)$ constructed when $v \leq v_{\min }$. 


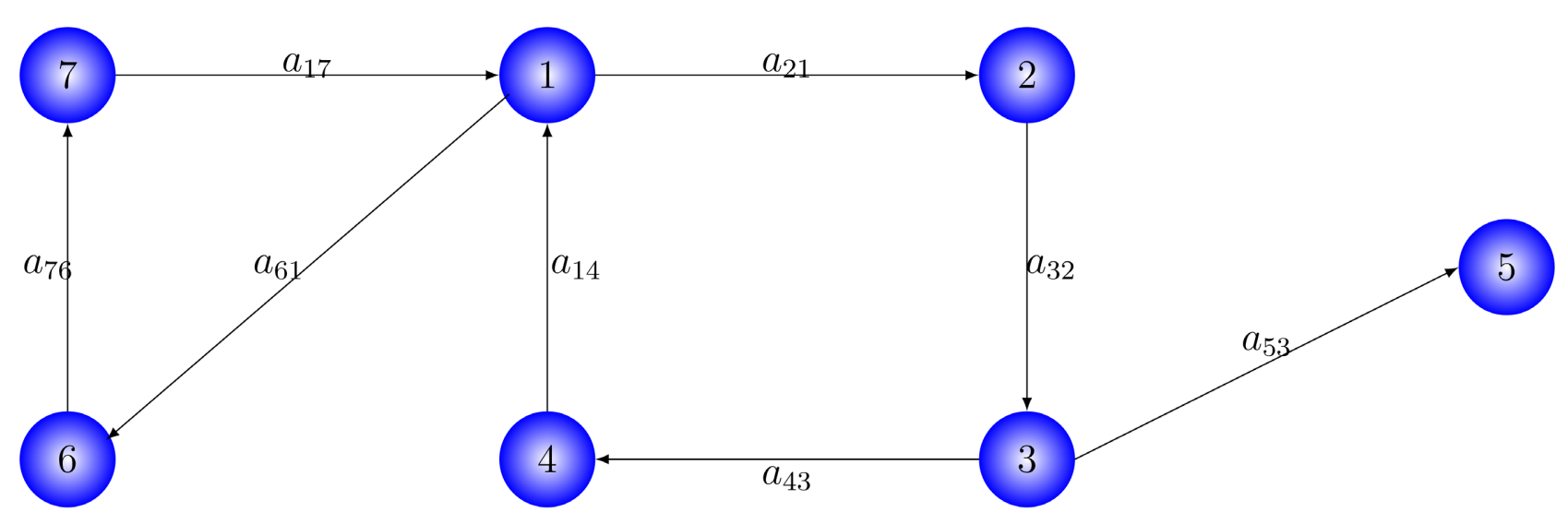

Figure 5. The weighted associated digraph $(G, A)$ constructed when $v>v_{\text {min }}$.

$a_{i j}=0$ such that the weighted matrix is $A=\left[a_{i j}\right]_{7 \times 7}$, where $a_{i j}>0$, (the weight of arc $(j, i))$. From this digraph, the first condition of Theorem 3.5 of [24] is satisfied. Let us check for the second condition of the cited Theorem. Along the two directed cycles, we have: $G_{12}+G_{23}+G_{34}+G_{41}=0$ and $G_{16}+G_{67}+G_{71}=0$. The second condition of Theorem 3.5 of [24] is also satisfied. Therefore, by this cited Theorem, there exists $c_{i, i \in\{1,2,3,4,5,6,7\}}$ such that:

$$
L=C_{1} L_{1}+c_{2} L_{2}+C_{3} L_{3}+c_{4} L_{4}+C_{5} L_{5}+c_{6} L_{6}+c_{7} L_{7},
$$

is a Lyapunov function for system (10)-(16). The relations between $c_{i}$ can be derived from Theorems 3.3 and 3.4 of [24] as follow: $c_{1} a_{17}=c_{7} a_{76}, c_{7} a_{76}=c_{6} a_{61}$, $c_{1} a_{14}=c_{4} a_{43}, c_{2} a_{21}=c_{3} a_{32}$ and $c_{5}=0$. Therefore, the Lyapunov function $L$ defined in (50) becomes:

$$
L=c_{1} L_{1}+C_{2} L_{2}+c_{3} L_{3}+C_{4} L_{4}+C_{6} L_{6}+C_{7} L_{7},
$$

and $c_{1}=c_{6} a_{61} / a_{17}, c_{6}=c_{4} a_{43} a_{17} / a_{61} a_{14}$ and $c_{2}=c_{3} a_{32} / a_{21}$. Thus, the fact that $\dot{L}=c_{1} \dot{L}_{1}+c_{2} \dot{L}_{2}+c_{3} \dot{L}_{3}+c_{4} \dot{L}_{4}+c_{6} \dot{L}_{6}+c_{7} \dot{L}_{7} \leq 0$ implies $Y=Y^{*}$. Hence, the largest invariant set for system (10)-(16), where $\dot{L}=0$ is the singleton set $\left\{Y^{*}\right\}$. This proves therefore the uniqueness and global asymptotic stability of $Y^{*}$ in the interior of $\Omega$ provided that $\mathcal{R}_{0}(v)>1$.

\section{Numeric Results and Discussion}

In this section, we have done some numerical simulations to confirm our analytical analysis. All the data used have been taken in the literature and the ones for fuzzy variables are assumed according for the amount of parasites required for malaria transmission in a particular group of individuals. The parameters $\mu$ and $\mu_{m}$ have dimensions, human ${ }^{-1} \times$ day $^{-1}$ and mosquitoes ${ }^{-1} \times$ day $^{-1}$ respectively. The parameters $\sigma_{h}, \delta, \theta_{h}, \sigma_{m}, \delta_{m}$ have for dimensions, day ${ }^{-1}$. The life expectation of Congolese people is estimated to be 65 years, the mortality death rate is $1 /(65 \times 365) \approx 421 \times 10^{-7}$. The malaria reported data of DRC, estimates the malaria-induced death rate to be $285 \times 10^{-5}$ [33]. The fuzzy parameters $\beta_{h}(v)$ and $\gamma_{h}(v)$ depend on the amount of parasites $v$. The amount of parasites (parasitic viral load) in the blood is determined and expressed as "para- 
sites per microliter ( $\mu \mathrm{l})$ of blood" from the parasite density formula (DP) [16] below:

$$
\text { DP }(\text { parasites per microliter })=\frac{\text { Number of parasites counted } \times 8000}{\text { Number of leukocytes }} .
$$

The minimum amount of parasites in the blood for malaria transmission is estimated under 1000 parasites/ $\mu$ l, the medium amount is estimated between 1000 to 10,000 parasites/ $\mu \mathrm{l}$ and the high parasitemia is estimated to over 10,000 parasites/ $\mu \mathrm{l}$ [16]. In this paper, for a particular group of individuals chosen, we take $v_{\min }=1000$ parasites $/ \mu \mathrm{l}, v_{M}=20000$ parasites $/ \mu \mathrm{l}$ and

$v_{\max }=50000$ parasites/ $\mu$ l [15] [16] [34]. The natural death rate of mosquitoes is equal to $\mu_{m}=0.001426$ [12]. From there, the parasite-induced death rate should be less than $\mu_{m}$, we therefore assumed that $\delta_{m}$ is in order of $10^{-6}$. When the maximum amounts of parasites are reached in blood cells, the recover becomes uncertain and can be assumed in order $10^{-3}$. Due to the fact that all recovered individuals form malaria become susceptible, we assume the progression rate $\theta_{h}$ equal to 0.138 . Table 1 gives descriptions, baseline/value and references of parameters used in this paper.

The simulations are done with different amounts of parasites. We give first the trends of $S_{h}, E_{h}, I_{h}$ and $R_{h}$ for different amounts of parasites and the trends of $S_{m}, E_{m}$ and $I_{m}$ for different amounts of parasites. We give the trends of $S_{h}$ and $E_{h}$ for different amounts of parasites. We finally give the trends of $\mathcal{R}_{0}^{f}$ and $\mathcal{R}_{0}(v)$ for different amounts of parasites.

Situation on Figure 6 occurs when the amount of parasites $v$ is less than the minimum amount required for malaria transmission $v_{\min }$. This assures the

Table 1. Parameters descriptions, their estimated values and references.

\begin{tabular}{clcc}
\hline Parameters & \multicolumn{1}{c}{ Descriptions } & Baseline/values & references \\
\hline$\beta_{h}(v)$ & $\begin{array}{l}\text { fuzzy contact transmission rate } \\
\text { of mosquitoes to humans }\end{array}$ & - & - \\
$\gamma_{h}(v)$ & fuzzy recovery rate of humans & - & - \\
$\mu$ & natural death rate of humans & $421 \times 10^{-7}$ & computed \\
$\delta_{m}$ & parasite-induced death rate & $10^{-6}$ & assumed \\
$\theta_{h}$ & $\begin{array}{l}\text { progression rate from } R_{h} \text { to } S_{h} \\
\gamma_{0}\end{array} \quad \begin{array}{l}\text { recovery rate of humans when } \\
\sigma_{m}\end{array} \quad \begin{array}{l}\text { latent rate of mosquitoes } \\
\mu_{m}\end{array} \quad \begin{array}{l}\text { natural death rate of mosquitoes } \\
\delta\end{array}$ malaria-induced death rate & $10^{-3}$ & assumed \\
$\sigma_{h}$ & latent rate of humans & 0.0769 & {$[3]$} \\
$\beta_{m}$ & $\begin{array}{l}\text { contact transmission rate of } \\
\text { humans to mosquitoes }\end{array}$ & $285 \times 10^{-5}$ & {$[33]$} \\
\hline
\end{tabular}


global stability of the disease-free equilibrium of the model (See Theorem (3)). Figure 7 and Figure 8 show the behaviors of the model when the amount of parasites $v$ is greater than the minimum amount required for malaria transmission $\left(v_{\min }<v<v_{M}\right.$ or $\left.v_{M} \leq v \leq v_{\max }\right)$. In addition, the basic reproduction number is greater than the unity $\mathcal{R}_{0}(v)>1$ in either case. These confirm the assumptions of Theorem (4). Either case, the endemic equilibrium is reached. See Theorem (4). Figure 9 shows the trends of susceptible humans for different amounts of parasites $v$. When the amounts of parasites in the body are less than the minimum amounts required, the population of susceptible humans is almost unchanged. When the amounts of parasites are greater than the minimum amounts required, the population of susceptible humans is decreasing.

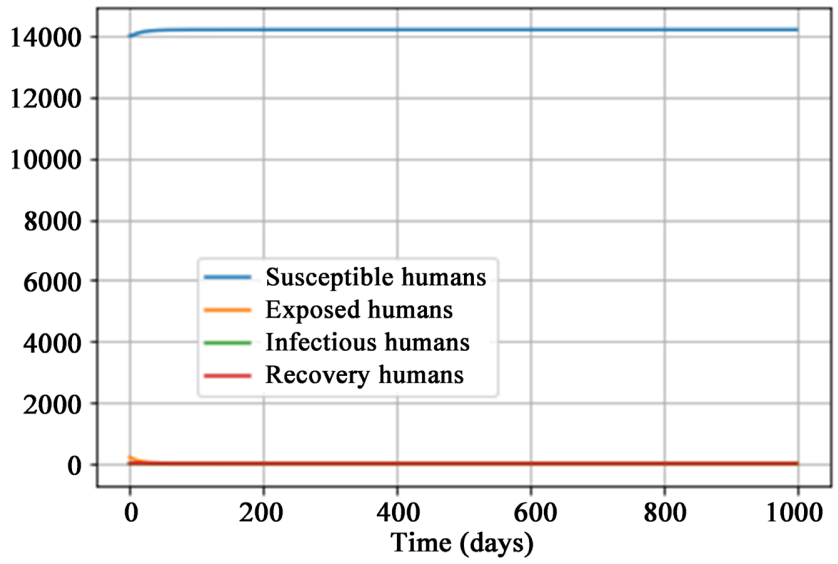

(a)

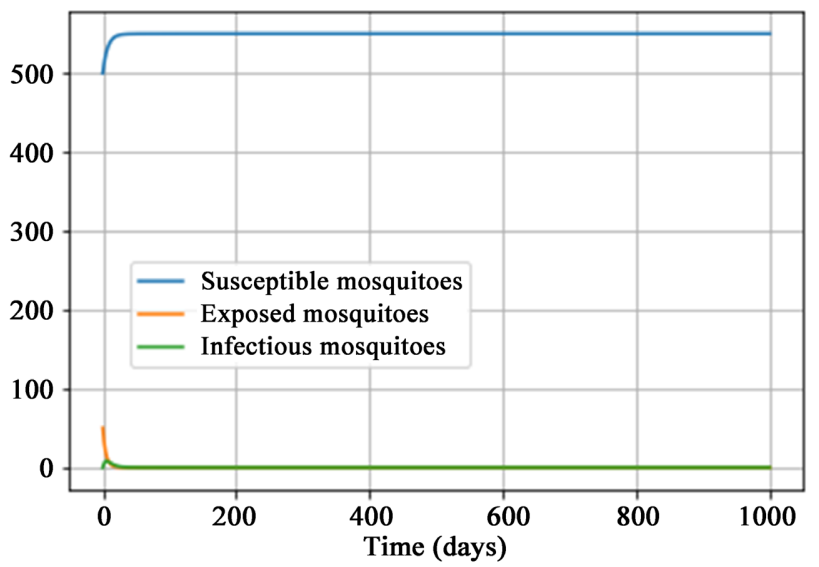

(b)

Figure 6. Distribution of individuals in each compartment for the parameter values given in Table 1 and for $v=100$ parasites with initial conditions $S_{h 0}=14000, E_{h 0}=100, I_{h 0}=100, R_{h 0}=0, S_{m 0}=500, E_{m 0}=10$ and $I_{m 0}=50$. We obtain $\mathcal{R}_{0}(v)=0<1$. The estimated disease-free equilibrium point is $\left(S_{h}^{0}, E_{h}^{0}, I_{h}^{0}, R_{h}^{0}, S_{m}^{0}, E_{m}^{0}, I_{m}^{0}\right)=(14200,0,0,0,560,0,0)$. (a) Distribution of humans population when $v \leq v_{\min }$; (b) Distribution of mosquitoes population when $v \leq v_{\min }$.

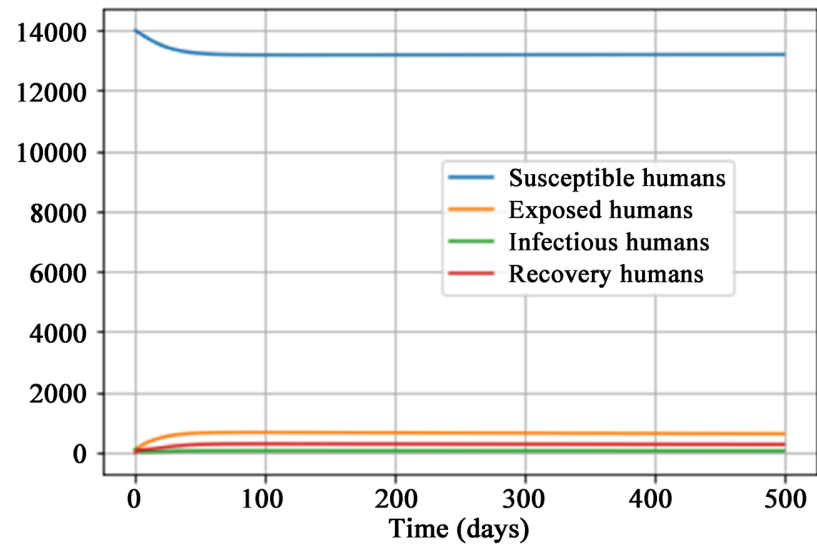

(a)

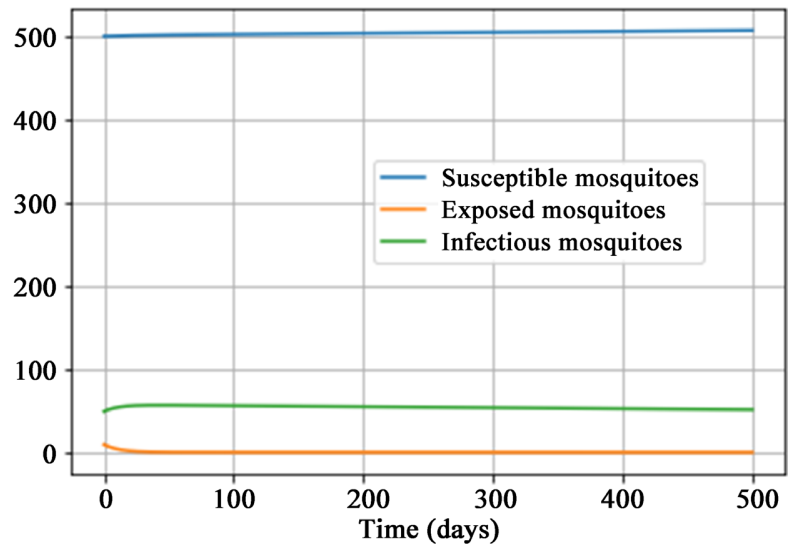

(b)

Figure 7. Distribution of individuals in each compartment for parameter values given in Table 1 and for $v=15000$ parasites with initials conditions $S_{h 0}=14000, E_{h 0}=100, I_{h 0}=100, R_{h 0}=0, S_{m 0}=500, E_{m 0}=10$ and $I_{m 0}=50$. We obtain $\mathcal{R}_{0}(v)=1.00074>1$. The estimated endemic equilibrium point is $\left(S_{h}^{*}, E_{h}^{*}, I_{h}^{*}, R_{h}^{*}, S_{m}^{*}, E_{m}^{*}, I_{m}^{*}\right)=(13100,810,55,130,502,3,53) .($ a) Distribution of humans population when $v_{\min }<v<v_{M}$; (b) Distribution of mosquitoes population when $v_{\min }<v<v_{M}$. 


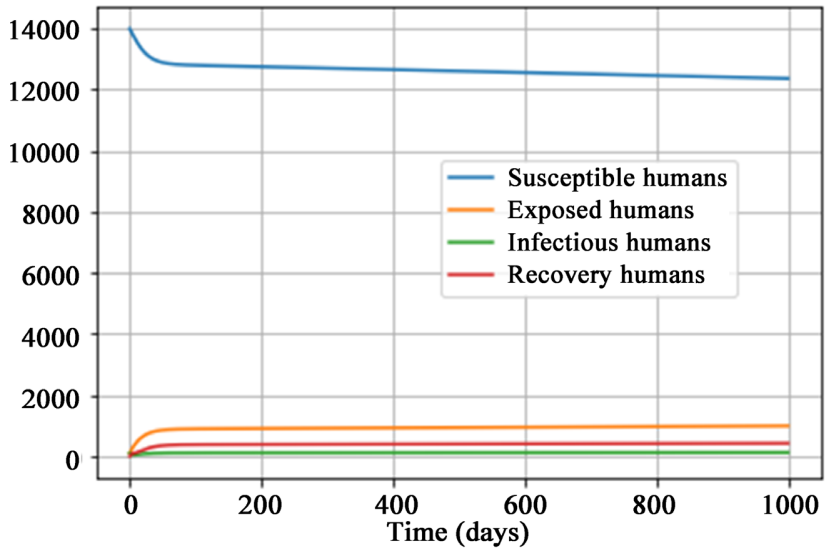

(a)

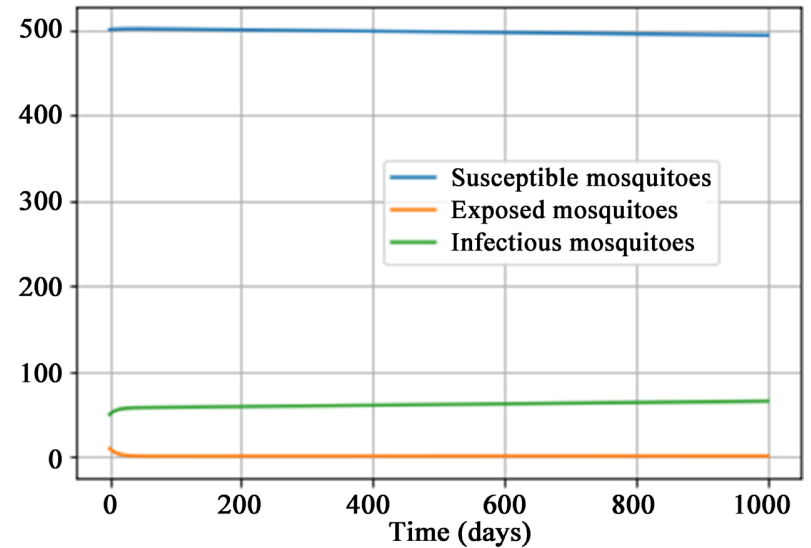

(b)

Figure 8. Distribution of individuals in each compartment for parameter values given in Table 1 and for $v=25000$ parasites with initials conditions $S_{h 0}=14000, E_{h 0}=100, I_{h 0}=100, R_{h 0}=0, S_{m 0}=500, E_{m 0}=10$ and $I_{m 0}=50$. We obtain $\mathcal{R}_{0}(v)=1.166>1$. The estimated endemic equilibrium point is $\left(S_{h}^{*}, E_{h}^{*}, I_{h}^{*}, R_{h}^{*}, S_{m}^{*}, E_{m}^{*}, I_{m}^{*}\right)=(12150,1000,155,230,495,2,60)$. (a) Distribution of humans population when $v_{M} \leq v \leq v_{\max }$; (b) Distribution of mosquitoes population when $v_{M} \leq v \leq v_{\max }$.

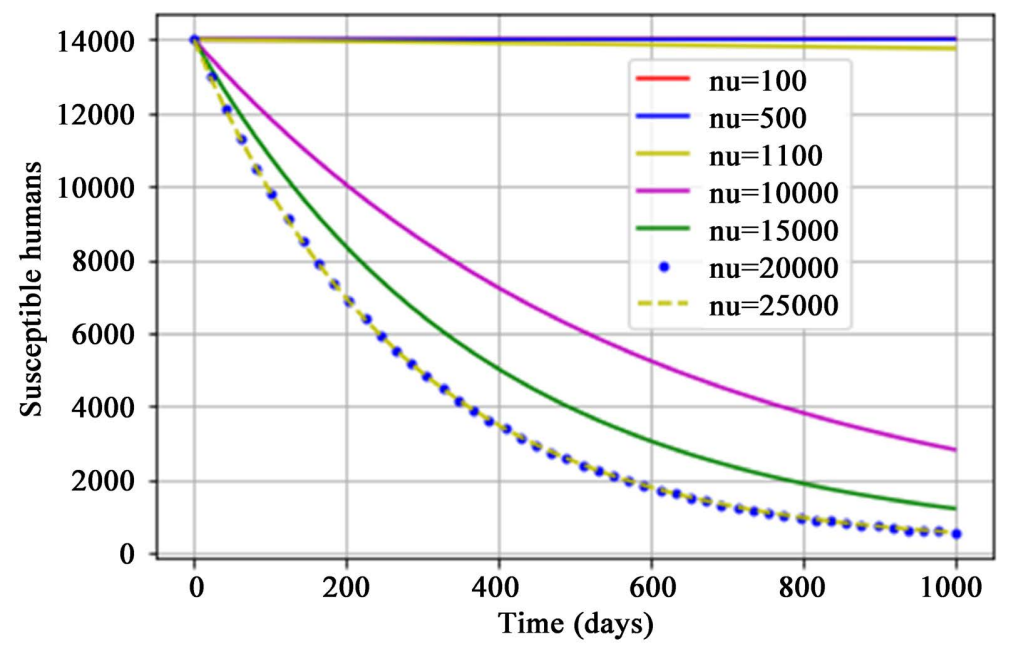

Figure 9. Behavior of susceptible humans for different amounts of parasites.

When the amounts of parasites approaches the maximum amount, the susceptible humans population goes to zero; which is normal because the transmission rate is approaching one. The case of exposed humans is quite different, (see Figure 10).

Figure 10 shows the behavior of exposed humans for different amounts of parasites $v$. When the amounts of parasites is less than the minimum amounts required $v_{\min }$, which implies $\beta_{h}(v)=0$, the population of exposed humans goes to zero. That means there is no contamination. Just when the amount of parasites exceeds the minimum amounts required, the population of exposed humans grows. Figure 11 shows the profiles of fuzzy contact rate $\beta_{h}(v)$ and fuzzy recovery rate $\gamma_{h}(v)$ for different amounts of parasites.

For the amount of parasites between $v_{\min }$ and $v_{M}$, the contact rate increases straightly as the amount of parasites $v$ is increasing. On the other hand, the 
recovery rate decreases straightly as the amount of parasites $v$ is increasing. When the amounts of parasites $v$ goes to zero, the recovery is almost possible, that is $\gamma_{h}(v)$ goes to 1 and when $v$ increases forever, the recovery is almost impossible, that is $\gamma_{h}(v)$ goes to zero. Finally, Figure 12 gives the trends of the basic reproduction number $\mathcal{R}_{0}(v)$ and the fuzzy basic reproduction number $\mathcal{R}_{0}^{f}$ for different amounts of parasites $v$.

The basic reproduction number is increasing when the amounts of parasites are increasing and vice-versa. When this amount approaches $v_{M}$, the basic reproduction number $\mathcal{R}_{0}(v)$ exceeds unity although the fuzzy basic reproduction number $\mathcal{R}_{0}^{f}$ remains less than the unity. The fuzzy basic reproduction number $\mathcal{R}_{0}^{f}$ exceeds unity when the amounts of parasites exceeds $v_{M}$.

\section{Concluding Remarks}

In this paper, we have proposed and studied the SEIRS-SEI model for malaria

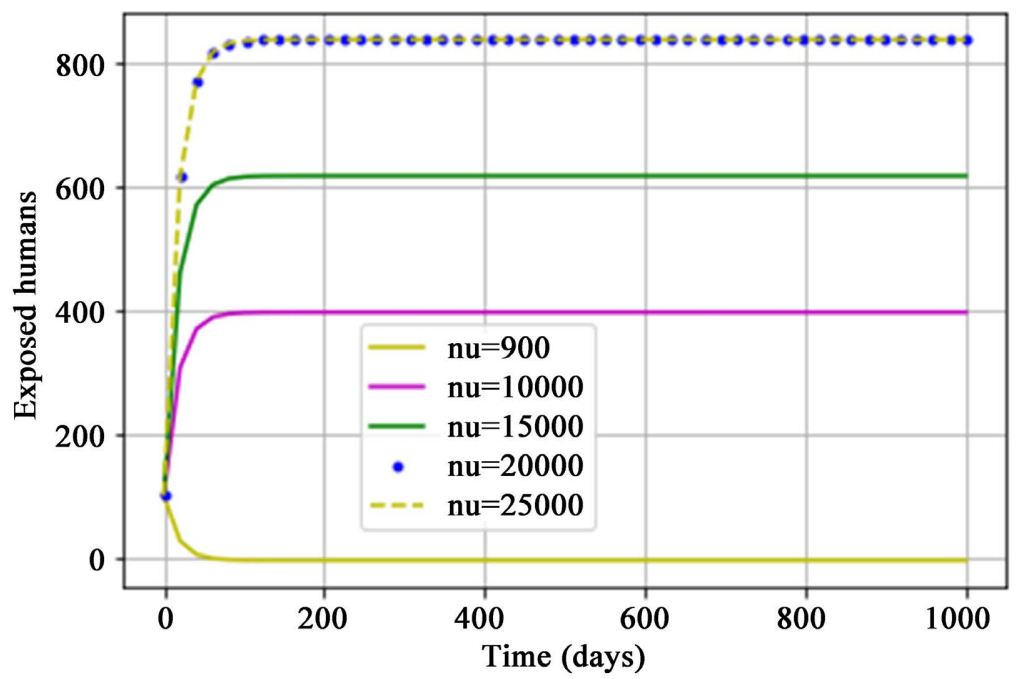

Figure 10. Behavior of exposed humans for different amount of parasites.

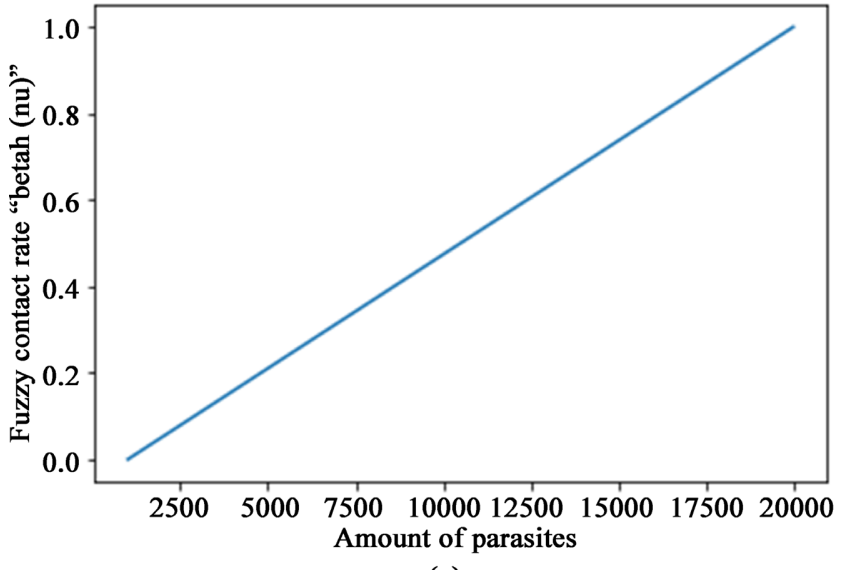

(a)

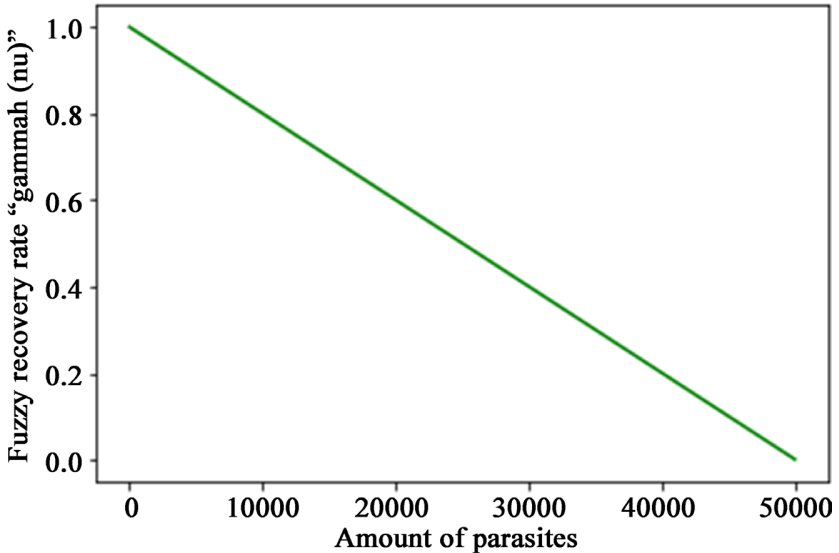

(b)

Figure 11. Distributions of $\beta_{h}(v)$ and $\gamma_{h}(v)$ for different amount of parasites. (a) Trends of $\beta_{h}(v)$ for $v_{\min }<v<v_{M}$; (b) Trends of $\gamma_{h}(v)$ for $0 \leq v \leq v_{\max }$. 


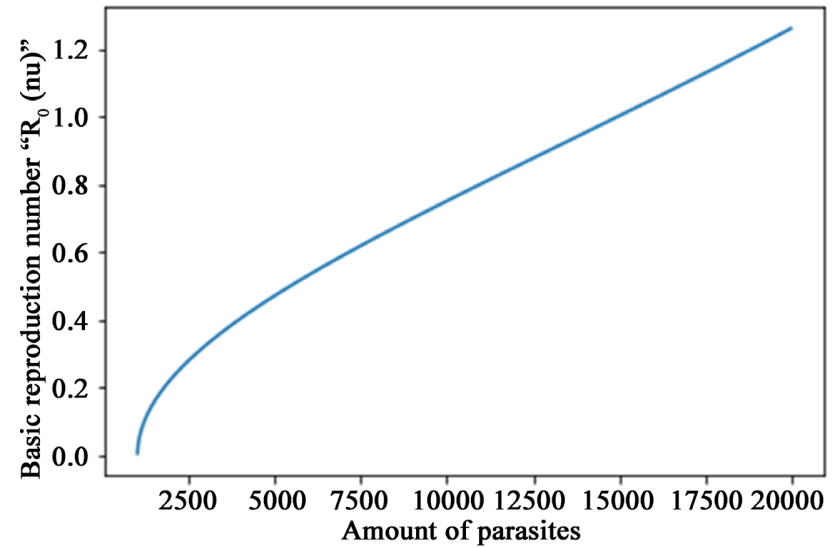

(a)

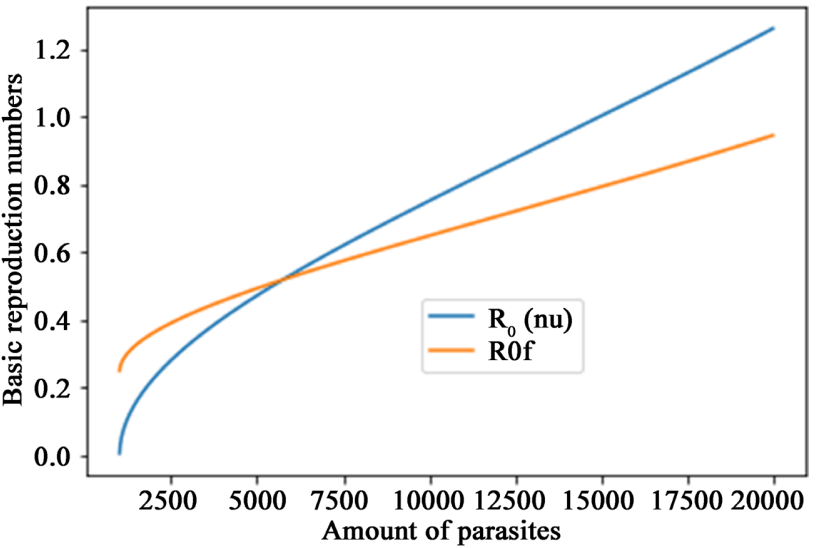

(b)

Figure 12. In the left hand side, the distribution of the basic reproduction number $\mathcal{R}_{0}(v)$ for different amounts of parasites and in the right hand side, distributions of the basic reproduction number $\mathcal{R}_{0}(v)$ and of the fuzzy basic reproduction number $\mathcal{R}_{0}^{f}$. (a) Trends of $\mathcal{R}_{0}(v)$ for $v_{\min }<v<v_{M}$; (b) Trends of $\mathcal{R}_{0}(v)$ and $\mathcal{R}_{0}^{f}$ for $v_{\min }<v<v_{M}$.

transmission dynamics in fuzzy environment. The fuzzy set theory used has allowed us to study the behavior of malaria transmission as depending on the amounts of parasites (parasitic viral load) in the body without dividing compartments into many other compartments. We considered the transmission rate $\beta_{h}(v)$ and the recovery rate $\gamma_{h}(v)$ as fuzzy variables depending on the amounts of parasites in the body $v$. To make the model more realistic, we studied a model in a fuzzy particular group $\mathrm{V}$ of individuals, which has a triangular membership function.

We introduced the fuzzy analysis of equilibrium of the fuzzy model by considering the amounts of parasites in the body. From this analysis, we derived the disease-free and endemic equilibriums of the fuzzy model. We proved that if the amounts of parasites in the body are less than the minimum amounts of parasites $v_{\min }$ required for malaria transmission, we reach the disease-free equilibrium. That is, no malaria in the population. We reach the endemic equilibrium, when the amounts of parasites in the body are greater than the minimum amounts of parasites required for malaria transmission. By the use of the classical Next Generation Matrix method as explained in [31], we expressed the basic reproduction number as a function of the amount of parasites $\mathcal{R}_{0}(v)$. A defuzzification procedure, such as "expected of a fuzzy variable", is used to derive and propose the "fuzzy basic reproduction number" $\mathcal{R}_{0}^{f}$.

We used the classical matrix-theoretical method as explained in [24] to introduce the fuzzy global stability of the disease-free equilibrium. We proved that when $v \leq v_{\text {min }}$ or $\mathcal{R}_{0}(v) \leq 1$, the disease-free equilibrium is global asymptotically stable and when $v>v_{\text {min }}$ and $\mathcal{R}_{0}(v)>1$, the disease-free equilibrium is unstable and there exists an endemic equilibrium point in the interior of the feasible set $\Omega$. Also by the use of the classical graph-theoretical method as explained in [24], we introduced the fuzzy global stability of the endemic equilibrium. We proved that the endemic equilibrium is globally asymptotically stable 
in the interior of the positively-invariant compact set $\Omega$ when the amounts of parasites in the body are greater than the minimum amounts required $\left(v>v_{\min }\right)$. The numerical simulations have been done for $v_{\min }=1000$ parasites $/ \mu \mathrm{l}$, $v_{M}=20000$ parasites $/ \mu \mathrm{l}$ and $v_{\max }=50000$ parasites $/ \mu \mathrm{l}$; and show that when the amounts of parasites increases, the susceptible humans and the exposed humans populations decrease and increase respectively.

Regarding in the analysis of this paper, the fuzzy models are more realistic, flexible, general than classical models because many of the analysis in the classical models can be derived from the fuzzy analysis. For instance, we obtain the disease-free equilibrium by the fuzzy analysis of the equilibrium of fuzzy model. That is, by analyzing the amounts of parasites in the body. In addition, in the classical model, the basic reproduction number is only a function of parameters, whereas in fuzzy environment, it's a function of the amounts of parasites. The fuzzy parameters used in this paper play an important role in the analysis of malaria transmission dynamics. We may extend this model by considering other parameters as fuzzy variables. One may consider, the mosquitoes population as a fuzzy variable.

\section{Data Availability}

The data used are available in the literature.

\section{Acknowledgements}

Authors gratefully thank the anonymous referees for the pertinent comments provided to improve the quality of this paper and the handling editor for their valuable comments and suggestions.

\section{Conflicts of Interest}

The authors declare no conflicts of interest regarding the publication of this paper.

\section{References}

[1] World Health Report (2020) Rapport 2020 sur le paludisme dans le monde. Messages généraux, 30 novembre 2020.

[2] Guide pratique de lutte contre le paludisme en enterprise (2018) Programme National de Lutte contre le Paludisme-SENEGAL.

[3] Roll back malaria (2010) Modélisation mathématique pour le contrôle et l'élimination du paludisme. Collection Progrès et Impact, novembre 2010.

[4] Bhuju, G., Phaijoo, G.R. and Gurung, D.B. (2020) Fuzzy Approach Analyzing SEIR-SEI Dengue Dynamics. BioMed Research International, 2020, Article ID: 1508613. https://doi.org/10.1155/2020/1508613

[5] de Barros, L.C., Ferreira Leite, M.B. and Bassanezi, R.C. (2003) The SI Epidemiological Models with a Fuzzy Transmission Parameter. Computers \& Mathematics with Applications, 45, 1619-1628. https://doi.org/10.1016/S0898-1221(03)00141-X

[6] Ross, R. (1910) The Preventive of Malaria. E.P. Dutton and Company, New York. 
[7] Agusto, F.B., Gumel, A. and Parham, P.E. (2015) Qualitative Assessment of the Role of Temperature Variations on Malaria Transmission Dynamics. Journal of Biological Dynamics, 23, Article ID: 1550030. https://doi.org/10.1142/S0218339015500308

[8] Djidjou-Demasse, R., Abiodun, G.J., Adeola, A.M. and Botai, J.O. (2020) Development and Analysis of a Malaria Transmission Mathematical Model with Seasonal Mosquitoe Life-History Traits. Studies in Applied Mathematics, 144, 389-411. https://doi.org/10.1111/sapm.12296

[9] Li, J., Welch, R.M., Nair, U.S., Sever, T.L., Irwin, D.E., Cordon-Rosales, C. and Padilla, N. (2002) Dynamic Malaria Models with Environmental Changes. Proceedings of 34th Southeastern Symposium on System Theory, Huntsville, 19 March 2002, 396-400. https://doi.org/10.1109/SSST.2002.1027075

[10] Nwankwo, A. and Okuonghae, D. (2019) Mathematical Assessment of the Impact of Different Microclimate Conditions on Malaria Transition Dynamics. Mathematical Biosciences and Engineering, 16, 1414-1444. https://doi.org/10.3934/mbe.2019069

[11] Traoré, B., Sangaré, B. and Traoré, S. (2017) A Mathematical Model of Malaria Transmission with Structured Vector Population and Seasonality. Journal of Applied Mathematics, 2017, Article ID 6754097. https://doi.org/10.1155/2017/6754097

[12] Huo, H.-F. and Qiu, G.-M. (2014) Stability of a Mathematical Model of Malaria Transmission with Relapse. Abstract and Applied Analysis, 2012, Article ID 289349. https://doi.org/10.1155/2014/289349

[13] Li, J., Zhao, Y. and Li, S. (2013) Fast and Slow Dynamics of Malaria Model with Relapse. Mathematical Biosciences, 246, 94-104.

https://doi.org/10.1016/j.mbs.2013.08.004

[14] Smith, H.L., Li, M.Y. and Wang, L.C. (2001) Global Dynamics of an SEIR Epidemic Model with Vertical Transmission. SIAM Journal on Applied Mathematics, 62, 58-69. https://doi.org/10.1137/S0036139999359860

[15] WHO (World Health Organization) (2010) Who Basic Malaria Microscopy. 2nd Edition. Part 1: Learner's Guide. World Health Organization, Geneva.

[16] Franke, M.F., Spiegelman, D., Ezeamama, A., Aboud, S., Msamanga, G.I., Mehta, S. and Fawzi, W.W. (2010) Malaria Parasitemia and CD4 T Cell Count, Viral Load, and Adverse HIV Outcomes among HIV-Infected Pregnant Women in Tanzania. The American Journal of Tropical Medicine and Hygiene, 82, 556-562. https://doi.org/10.4269/ajtmh.2010.09-0477

[17] Zadeh, L.A. (1965) Fuzzy Sets. Information and Control, 8, 338-353. https://doi.org/10.1016/S0019-9958(65)90241-X

[18] Jain, M., Sharma, G.C. and Kumar Sharma, S. (2006) Transmission Dynamics of Malaria in Human Host: A Neuro-Fuzzy Approach. IJE Transactions A: Basics, 19, 35-48.

[19] Zarei, H., Vahidian Kamyad, A. and Akbar Heydari, A. (2012) Fuzzy Modeling and Control of HIV Infection. Computational and Mathematical Methods in Medecine, 2012, Article ID: 893474. https://doi.org/10.1155/2012/893474

[20] Mondal, P.K., Jana, S., Haldar, P. and Kar, T.K. (2015) Dynamical Behavior of an Epidemic Model in a Fuzzy Transmission. International Journal of Uncertainty, Fuzziness and Knowledge-Based Systems, 23, 651-665. https://doi.org/10.1142/S0218488515500282

[21] Emokhare, B.O. and Igbape, E.M. (2015) Fuzzy Logic Based Approach to Early Diagnosis of Ebola Hemorrhagic Fever. Proceedings of the World Congress on Engineering and Computer Science, Vol. 2, San Francisco, 21-23 October 2015.

[22] Verma, R., Tiwari, S.P. and Kumar Upadhyay, R. (2018) Dynamical Behaviors of 
Fuzzy SIR Epidemic Model. Proceedings of the Conference of the European Society for Fuzzy Logic and Technology, Warsaw, 13-15 September 2017, 482-492. https://doi.org/10.1007/978-3-319-66827-7 45

[23] Liu, B. and Liu, Y.-K. (2002) Expected Value of Fuzzy Variable and Fuzzy Expected Value Models. IEEE Transactions on Fuzzy Systems, 10, 445-450.

https://doi.org/10.1109/TFUZZ.2002.800692

[24] Shuai, Z. and van den Driessche, P. (2013) Global Stability of Infectious Disease Models Using Lyapunov Functions. SIAM Journal on Applied Mathematics, 73, 1513-1532. https://doi.org/10.1137/120876642

[25] Kampempe, J.D.B. and Luhandjula, M.K. (2012) Chance-Constrained Approaches for Multiobjective Stochastic Linear Programming Problems. American Journal of Operations Research, 2, 519-526. https://doi.org/10.4236/ajor.2012.24061

[26] Lei, C., Ji, H. and Hu, N. (2018) Reliability Analysis of Automatic Transmission Based on T-S Fuzzy Fault Tree. Open Access Library Journal, 5, 1-13. https://doi.org/10.4236/oalib.1104659

[27] Zadeh, L.A. (1978) Fuzzy Sets as a Basis for a Theory of Possibility. Fuzzy Sets and Systems, 1, 3-28. https://doi.org/10.1016/0165-0114(78)90029-5

[28] Liu, B. (2007) Uncertainty Theory. Vol. 15, 2nd Edition, Springer-Verlag, Berlin.

[29] Liu, B. (2015) Uncertainty Theory. 4th Edition Springer-Verlag, Berlin. https://doi.org/10.1007/978-3-662-44354-5

[30] Wiggins, S. (2003) Introduction to Applied Nonlinear Dynamical Systems and Chaos. Vol. 2, Springer, New York.

[31] van den Driessche, P. and Watmough, J. (2002) Reproduction Numbers and Sub-Threshold Endemic Equilibria for Compartmental Models of Disease Transmission. Mathematical Biosciences, 180, 29-48.

https://doi.org/10.1016/S0025-5564(02)00108-6

[32] LaSalle, J.P. (1976) The Stability of Dynamical Systems. Society for Industrial and Applied Mathematics, University City, 5.

[33] Ministère de la Santé Publique of the Democratic Republic of Congo (2007) Faire reculer le paludisme: Plan stratégique 2007-2011. RDC, Ministère de la Santé Publique, Programme National de Lutte contre le Paludisme (PNLP), Juin 2007.

[34] Sciensano (2018) Comptage de parasites plasmodium dans le sang. Sciensano, Rue Juliette Wytsman 14.1050, Bruxelles.

[35] Olaniyi, S., Okosun, K.O., Adesanya, S.O. and Lebelo, R.S. (2020) Modelling Malaria Dynamics with Partial Immunity and Protected Travellers: Optimal Control and Cost Effectiveness Analysis. Journal of Biological Dynamics, 14, 90-115. https://doi.org/10.1080/17513758.2020.1722265 